TRANSACTIONS OF THE

AMERICAN MATHEMATICAL SOCIETY

Volume 359, Number 4, April 2007, Pages 1653-1683

S 0002-9947(06)03975-4

Article electronically published on September 19, 2006

\title{
ALGEBRAIC INDEPENDENCE IN THE GROTHENDIECK RING OF VARIETIES
}

\author{
N. NAUMANN
}

\begin{abstract}
We give sufficient cohomological criteria for the classes of given varieties over a field $k$ to be algebraically independent in the Grothendieck ring of varieties over $k$ and construct some examples.
\end{abstract}

\section{INTRODUCTION}

Let $k$ be a field. The Grothendieck ring of varieties over $k$, denoted $K_{0}\left(\operatorname{Var}_{k}\right)$, was defined by A. Grothendieck in $\mathrm{G}$.

The abelian group $K_{0}\left(\operatorname{Var}_{k}\right)$ is generated by the isomorphism classes $[X]$ of separated $k$-schemes of finite type $X / k$ subject to the relations $[X]=[Y]+[X-Y]$ for $Y \subset X$ a closed subscheme. Multiplication is given by $\left[X_{1}\right] \cdot\left[X_{2}\right]:=\left[X_{1} \times_{k}\right.$ $X_{2}$ ] and makes $K_{0}\left(\operatorname{Var}_{k}\right)$ a commutative ring with unit [Spec$\left.(k)\right]$. By its very definition $K_{0}\left(\operatorname{Var}_{k}\right)$ is the value group of the universal Euler-Poincaré characteristic with compact support for varieties over $k$ and is thus a fundamental invariant of the algebraic geometry over $k$. The most outstanding result on the structure of $K_{0}\left(\operatorname{Var}_{k}\right)$ for $k$ of characteristic zero is a presentation of the ring $K_{0}\left(\operatorname{Var}_{k}\right)$ in terms of generators and relations anticipated by E. Looijenga and proved by his student F. Bittner [Bi]. B. Poonen showed that for $k$ of characteristic zero a linear combination of the classes of suitable abelian varieties is a zero divisor in $K_{0}\left(\operatorname{Var}_{k}\right)$ P , and J. Kollár has computed the subring of $K_{0}\left(\operatorname{Var}_{k}\right)$ generated by the classes of conics for suitable base fields $k[\mathrm{Ko}$. A deep problem pertaining to the structure of $K_{0}\left(\operatorname{Var}_{k}\right)$ is the rationality of motivic zeta-functions posed by M. Kapranov [ $\mathrm{K}$ ] on which there has been recent progress due to M. Larsen and V. Lunts [LL1]. This problem is intimately related to the finite-dimensionality of motives; cf. A for an exposition. Furthermore, the ring $K_{0}\left(\operatorname{Var}_{k}\right)$ plays a central rôle in the theory of motivic integration; $\mathrm{cf}$. [LO. The present note is dedicated to the following problem about the structure of $K_{0}\left(\operatorname{Var}_{k}\right)$.

Given varieties $X_{i} / k$, when are their classes $\left[X_{i}\right] \in K_{0}\left(\operatorname{Var}_{k}\right)$ algebraically independent (in the sense made precise at the end of this Introduction)?

We give a number of sufficient cohomological conditions for this to be the case and construct some examples. Our main results are also valid in positive characteristic where there is no known Theorem about the structure of $K_{0}\left(\operatorname{Var}_{k}\right)$. We now review the content of the individual sections.

Received by the editors July 28, 2004 and, in revised form, January 20, 2005.

2000 Mathematics Subject Classification. Primary 14A10.

Key words and phrases. Grothendieck ring of varieties, motivic measure.

(C)2006 American Mathematical Society Reverts to public domain 28 years from publication 
In section 2 we construct various ring homomorphisms $\mu_{k}: K_{0}\left(\operatorname{Var}_{k}\right) \longrightarrow R$ (so called motivic measures) all based on étale cohomology. The weight filtration is used to show that the classes of proper smooth varieties over a finitely generated field $k$ are algebraically independent in $K_{0}\left(\operatorname{Var}_{k}\right)$ as soon as their first étale cohomology groups are algebraically independent in the ring of virtual $l$-adic Galois representations (Corollary 3). We then reformulate known properties of rationality and $l$-independence for varieties over $\mathbb{F}_{q}$ (Proposition 7 ).

In Subsection 3.1 we reduce the problem of algebraic independence of virtual $l$-adic Galois representations to a problem about representations of (possibly nonconnected) reductive groups, give a result illustrating the subtleties arising from this non-connectedness (Theorem 11) and construct an infinite sequence of curves over a given finite field $k$ the classes of which are algebraically independent in $K_{0}\left(\operatorname{Var}_{k}\right)$ (Theorems 12 and 17). This shows in particular that $K_{0}\left(\operatorname{Var}_{k}\right)$ contains a polynomial ring in infinitely many variables and supports the intuition that $K_{0}\left(\operatorname{Var}_{k}\right)$ should be a large ring. We obtain a similar result for number fields using only elliptic curves (Theorem 13).

In Subsection 3.2 we use a lemma of Skolem together with the aforementioned rationality properties to give another approach to the algebraic independence of virtual $l$-adic Galois representations and show that a generic pair of curves over a finite field has algebraically independent classes in the Grothendieck ring of varieties (Theorem 26 in Subsection 3.5). We also determine the structure of the subring of $K_{0}\left(\operatorname{Var}_{\mathbb{F}_{q}}\right)$ generated by zero-dimensional varieties showing in particular that $K_{0}\left(\operatorname{Var}_{\mathbb{F}_{q}}\right)$ contains infinitely many zero divisors (Theorem 25).

The following definition will be central throughout the rest of this note.

Definition 1. Let $R$ be a commutative unitary ring and let $n \mathbb{Z}$ be the kernel of $\mathbb{Z} \longrightarrow R$. We call a set $\left\{x_{i}: i \in I\right\}$ of elements $x_{i} \in R$ algebraically independent if the morphism

$$
(\mathbb{Z} / n)\left[T_{i}: i \in I\right] \longrightarrow R, T_{i} \mapsto x_{i}
$$

is injective.

If $R$ is a field, then this means algebraically independent over the prime field of $R$ in the usual sense. Note that $1 / 2 \in \mathbb{Q}$ is algebraically dependent though not integral over $\mathbb{Z}$; cf. Theorem 24. All the rings appearing below will have $n=0$, i.e. $\mathbb{Z} \subset R$. In this case, elements $x_{i} \in R$ are algebraically independent if and only if the subring of $R$ they generate is a polynomial ring over $\mathbb{Z}$ in the variables $x_{i}$.

\section{A motivic measure}

2.1. General construction. Let $k$ be a field. We fix a separable closure $\bar{k}$ of $k$ and a rational prime $l$ different from the characteristic of $k$. For $X / k$ separated and of finite type we denote by

$$
H_{c}^{i}(\bar{X}):=H_{c}^{i}\left(X \times_{k} \bar{k}, \mathbb{Q}_{l}\right) \text { for } i \geq 0
$$

the étale cohomology with compact support and constant coefficients $\mathbb{Q}_{l}$ of the base change $X \times{ }_{k} \bar{k}$. Then $H_{c}^{i}(\bar{X})$ is naturally a $G_{k}:=\operatorname{Aut}(\bar{k} / k)$-module, and denoting by $\operatorname{Rep}_{G_{k}} \mathbb{Q}_{l}$ the category of finite-dimensional continuous representations of $G_{k}$ over $\mathbb{Q}_{l}$ (i.e. $l$-adic Galois representations) we have a motivic measure

$$
\mu_{k}: K_{0}\left(\operatorname{Var}_{k}\right) \longrightarrow K_{0}\left(\operatorname{Rep}_{G_{k}} \mathbb{Q}_{l}\right),[X] \mapsto \sum_{i}(-1)^{i}\left[H_{c}^{i}(\bar{X})\right]
$$


In fact, (1) is (well defined and) a homomorphism of abelian groups by excision, multiplicative by the Künneth formula and clearly preserves the unit.

For a finite extension $k \subset L \subset \bar{k}$ we have a ring homomorphism (base change)

$$
-\times_{k} L: K_{0}\left(\operatorname{Var}_{k}\right) \longrightarrow K_{0}\left(\operatorname{Var}_{L}\right),[X] \mapsto\left[X \times_{k} L\right]
$$

and a homomorphism of abelian groups (restriction of scalars)

$$
/ k: K_{0}\left(\operatorname{Var}_{L}\right) \longrightarrow K_{0}\left(\operatorname{Var}_{k}\right),[X \longrightarrow \operatorname{Spec}(L)] \mapsto[X \longrightarrow \operatorname{Spec}(L) \longrightarrow \operatorname{Spec}(k)] \text {. }
$$

From the inclusion $G_{L} \subset G_{k}$ we have an exact tensor functor (restriction)

$$
\operatorname{Res}_{G_{L}}^{G_{k}}: \operatorname{Rep}_{G_{k}} \mathbb{Q}_{l} \longrightarrow \operatorname{Rep}_{G_{L}} \mathbb{Q}_{l}
$$

and an exact functor (induction)

$$
\operatorname{Ind}_{G_{L}}^{G_{k}}: \operatorname{Rep}_{G_{L}} \mathbb{Q}_{l} \longrightarrow \operatorname{Rep}_{G_{k}} \mathbb{Q}_{l}, V \mapsto \mathbb{Q}_{l}\left[G_{k}\right] \otimes_{\mathbb{Q}_{l}\left[G_{L}\right]} V
$$

inducing a ring homomorphism and a homomorphism of abelian groups on the level of $K_{0}$ to be denoted by the same symbols. The following diagram is commutative:

$$
\begin{array}{cc}
K_{0}\left(\operatorname{Var}_{k}\right) \stackrel{\mu_{k}}{\longrightarrow} K_{0}\left(\operatorname{Rep}_{G_{k}} \mathbb{Q}_{l}\right) \\
-\times_{k} L|\uparrow| / k & \operatorname{Res}_{G_{L}}^{G_{k}}|\uparrow| \\
K_{0}\left(\operatorname{Var}_{L}\right) \stackrel{\operatorname{Ind}_{G_{L}}^{G_{k}}}{\longrightarrow} & K_{0}\left(\operatorname{Rep}_{G_{L}} \mathbb{Q}_{l}\right)
\end{array}
$$

2.2. Weight filtration. Notation being as in Subsection 2.1 we now assume in addition that $k$ is finitely generated. In this subsection we will incorporate the weight filtration on $H_{c}^{*}(\bar{X})$ into the motivic measure (11). We need to assume that $k$ is finitely generated in order to have a theory of weights. Vaguely speaking, this is a substitute (valid in any characteristic) of Hodge theory and will allow us to isolate $H^{1}$ from the Euler-Poincaré characteristic in Theorem 2 below.

We refer the reader to [J], $\S 6$, for the definition of the full subcategory

$$
\mathrm{WRep}_{G_{k}} \mathbb{Q}_{l} \subset \operatorname{Rep}_{G_{k}} \mathbb{Q}_{l}
$$

of $l$-adic Galois representations having a weight filtration. From [J], Lemma 6.8.2 and (6.8.3), we know that $H_{c}^{i}(\bar{X}) \in \operatorname{WRep}_{G_{k}} \mathbb{Q}_{l}$ holds for all $X / k$ separated and of finite type and hence (1) factors as

$$
\mu_{k}: K_{0}\left(\operatorname{Var}_{k}\right) \longrightarrow K_{0}\left(\operatorname{WRep}_{G_{k}} \mathbb{Q}_{l}\right)
$$

For $V \in \operatorname{WRep}_{G_{k}} \mathbb{Q}_{l}$ we denote by $W \bullet V$ the weight filtration of $V$ and by $\operatorname{Gr}_{i}^{W}(V):=$ $W_{i} V / W_{i-1} V(i \in \mathbb{Z})$ the associated graded of weight $i$. As any morphism in $\mathrm{WRep}_{G_{k}} \mathbb{Q}_{l}$ is strictly compatible with the weight filtration ([J], Lemma 6.8.1), the functor

$$
\mathrm{Gr}_{i}^{W}: \operatorname{WRep}_{G_{k}} \mathbb{Q}_{l} \longrightarrow \operatorname{Rep}_{G_{k}} \mathbb{Q}_{l}, V \mapsto \operatorname{Gr}_{i}^{W}(V)
$$

is exact ([D1], Proposition 1.1.11, ii)) and thus induces a homomorphism

$$
\mathrm{Gr}_{i}^{W}: K_{0}\left(\mathrm{WRep}_{G_{k}} \mathbb{Q}_{l}\right) \longrightarrow K_{0}\left(\operatorname{Rep}_{G_{k}} \mathbb{Q}_{l}\right) .
$$

So we have a homomorphism of abelian groups

$$
\Phi: K_{0}\left(\operatorname{WRep}_{G_{k}} \mathbb{Q}_{l}\right) \longrightarrow K_{0}\left(\operatorname{Rep}_{G_{k}} \mathbb{Q}_{l}\right)\left[T, T^{-1}\right], x \mapsto \sum_{i \in \mathbb{Z}} \operatorname{Gr}_{i}^{W}(x) T^{i}
$$


which is a ring homomorphism as follows from

$$
\operatorname{Gr}_{i}^{W}(V \otimes W) \simeq \bigoplus_{a+b=i} \operatorname{Gr}_{a}^{W}(V) \otimes \operatorname{Gr}_{b}^{W}(W) \text { for } i \in \mathbb{Z} \text { and } V, W \in \operatorname{WRep}_{G_{k}} \mathbb{Q}_{l} \text {. }
$$

As $\operatorname{Gr}_{i}^{W}\left(H_{c}^{*}(\bar{X})\right)=0$ for $i<0$ (see [Ka]) we obtain, composing (44) and (5), a motivic measure

$$
\mu_{k}: K_{0}\left(\operatorname{Var}_{k}\right) \longrightarrow K_{0}\left(\operatorname{Rep}_{G_{k}} \mathbb{Q}_{l}\right)[T]
$$

given explicitly by

$$
\mu_{k}([X])=\sum_{i \geq 0}\left(\sum_{j}(-1)^{j}\left[\operatorname{Gr}_{i}^{W}\left(H_{c}^{j}(\bar{X})\right)\right]\right) T^{i} .
$$

The slight abuse of notation in denoting (11) and (6) by the same symbol $\mu_{k}$ will cause no confusion.

For a finite extension $k \subset L \subset \bar{k}$ we extend the morphisms induced on $K_{0}$ by (21) (resp. (32) ) to $K_{0}\left(\operatorname{Rep}_{G_{k}} \mathbb{Q}_{l}\right)[T]$ (resp. $K_{0}\left(\operatorname{Rep}_{G_{L}} \mathbb{Q}_{l}\right)[T]$ ) by demanding that $T \mapsto T$. With $\mu_{k}$ as in (6) we then have a commutative diagram

$$
\begin{aligned}
& K_{0}\left(\operatorname{Var}_{k}\right) \stackrel{\mu_{k}}{\longrightarrow} K_{0}\left(\operatorname{Rep}_{G_{k}} \mathbb{Q}_{l}\right)[T] \\
& -\times_{k} L \downarrow\left|/ k \quad \operatorname{Res}_{G_{L}}^{G_{k}} \downarrow\right| \operatorname{Ind}_{G_{L}}^{G_{k}} \\
& K_{0}\left(\operatorname{Var}_{L}\right) \stackrel{\mu_{L}}{\longrightarrow} K_{0}\left(\operatorname{Rep}_{G_{L}} \mathbb{Q}_{l}\right)[T]
\end{aligned}
$$

The commutativity of (8) follows from the compatibility of the weight filtration with restriction and induction, which we leave to the reader to verify using the uniqueness of the weight filtration ([J], Lemma 6.8.1, a)). We now use the weight filtration to establish the following criterion for algebraic independence in $K_{0}\left(\operatorname{Var}_{k}\right)$.

For $X / k$ separated and of finite type and $i \geq 0$ we write

$$
\operatorname{Gr}_{i}^{W}(X):=\sum_{j}(-1)^{j}\left[\operatorname{Gr}_{i}^{W}\left(H_{c}^{j}(\bar{X})\right)\right] \in K_{0}\left(\operatorname{Rep}_{G_{k}} \mathbb{Q}_{l}\right) .
$$

Then $\mu_{k}([X])=\sum_{i} \operatorname{Gr}_{i}^{W}(X) T^{i} \in K_{0}\left(\operatorname{Rep}_{G_{k}} \mathbb{Q}_{l}\right)[T]$; see (7).

Theorem 2. Let $k$ be finitely generated and let $X_{1}, \ldots, X_{n} / k$ be separated and of finite type and assume that $\operatorname{Gr}_{0}^{W}\left(X_{i}\right) \in \mathbb{Z} \subseteq K_{0}\left(\operatorname{Rep}_{G_{k}} \mathbb{Q}_{l}\right)$ for all $i$ and that the $\operatorname{Gr}_{1}^{W}\left(X_{i}\right)$ are algebraically independent in $K_{0}\left(\operatorname{Rep}_{G_{k}} \mathbb{Q}_{l}\right)$. Then the $\mu_{k}\left(\left[X_{i}\right]\right)$ are algebraically independent in $K_{0}\left(\operatorname{Rep}_{G_{k}} \mathbb{Q}_{l}\right)[T]$ and hence the $\left[X_{i}\right]$ are algebraically independent in $K_{0}\left(\operatorname{Var}_{k}\right)$.

Note that $K_{0}\left(\operatorname{Rep}_{G_{k}} \mathbb{Q}_{l}\right)$ is augmented over $\mathbb{Z}$ by the degree map, hence $\mathbb{Z} \subseteq$ $K_{0}\left(\operatorname{Rep}_{G_{k}} \mathbb{Q}_{l}\right)$. The last assertion of the Theorem follows from the existence of the motivic measure (6).

Corollary 3. Let $k$ be finitely generated and let $X_{1}, \ldots, X_{n} / k$ be proper and smooth. Then, if the $\left[H_{c}^{1}\left(\bar{X}_{i}\right)\right]$ are algebraically independent in $K_{0}\left(\operatorname{Rep}_{G_{k}} \mathbb{Q}_{l}\right)$, so are the $\mu_{k}\left(\left[X_{i}\right]\right)$ in $K_{0}\left(\operatorname{Rep}_{G_{k}} \mathbb{Q}_{l}\right)[T]$ and hence also the $\left[X_{i}\right]$ in $K_{0}\left(\operatorname{Var}_{k}\right)$.

Proof. As the $X_{i} / k$ are proper and smooth we have $\operatorname{Gr}_{0}^{W}\left(X_{i}\right)=\left[H_{c}^{0}\left(\bar{X}_{i}\right)\right]=$ $\left[\mathbb{Q}_{l}^{\oplus\left|\pi_{0}\left(\bar{X}_{i}\right)\right|}\right]=\left|\pi_{0}\left(\bar{X}_{i}\right)\right| \in \mathbb{Z}$ and $\operatorname{Gr}_{1}^{W}\left(X_{i}\right)=-\left[H_{c}^{1}\left(\bar{X}_{i}\right)\right]$. Since the $\left[H_{c}^{1}\left(\bar{X}_{i}\right)\right]$ are algebraically independent by assumption, so are the $\operatorname{Gr}_{1}^{W}\left(X_{i}\right)$, and Theorem 2 applies. 
For the proof of Theorem 2 we need some elementary preparation. Let $R$ be a commutative unitary ring. On $R\left[T_{1}, \ldots, T_{n}\right]$ we have the usual derivations $\frac{\partial}{\partial T_{i}}$, and for $\underline{\alpha}=\left(\alpha_{1}, \ldots, \alpha_{n}\right) \in \mathbb{N}_{0}^{n}$ we write $\frac{\partial^{|\underline{\alpha}|}}{\partial T_{1}^{\alpha_{1}} \ldots \partial T_{n}^{\alpha_{n}}}$ for their iterations; here $|\underline{\alpha}|:=\sum \alpha_{i}$.

Now let $f_{1}, \ldots, f_{n} \in R[T], G \in \mathbb{Z}\left[T_{1}, \ldots, T_{n}\right]$ be given and put $\tilde{G}(T)$ $:=G\left(f_{1}, \ldots, f_{n}\right) \in R[T]$. For $N \geq 1$ let $I_{N} \subseteq R[T]$ be the ideal generated by $\frac{\partial \mid \underline{|x|} G}{\partial T_{1}^{\alpha_{1}} \ldots \partial T_{n}^{\alpha_{n}}}\left(f_{1}, \ldots, f_{n}\right)$ for $|\underline{\alpha}| \leq N$ and put $I_{0}:=0$. The $\left(I_{N}\right)_{N \geq 0}$ form an ascending chain of ideals of $R[T]$ and $\frac{d}{d T}\left(I_{N}\right) \subset I_{N+1}$ for $N \geq 0$.

Proposition 4. For $N \geq 1$ we have

$$
\frac{d^{N} \tilde{G}}{d T^{N}} \equiv \sum_{1 \leq i_{1}, \ldots, i_{N} \leq n} \frac{\partial^{N} G}{\partial T_{i_{1}} \ldots \partial T_{i_{N}}}\left(f_{1}, \ldots, f_{n}\right) \frac{d f_{i_{1}}}{d T} \ldots \frac{d f_{i_{N}}}{d T}\left(\bmod I_{N-1}\right) .
$$

Proof. We use induction on $N \geq 1$. We have

$$
\frac{d \tilde{G}}{d T}=\frac{d}{d T} G\left(f_{1}, \ldots, f_{n}\right)=\sum_{1 \leq i_{1} \leq n} \frac{\partial G}{\partial T_{i_{1}}}\left(f_{1}, \ldots, f_{n}\right) \frac{d f_{i_{1}}}{d T},
$$

i.e. the result for $N=1$. We assume $N \geq 2$ and compute, using the induction hypothesis:

$$
\frac{d^{N} \tilde{G}}{d T^{N}}=\frac{d}{d T}\left(\sum_{1 \leq i_{1}, \ldots, i_{N-1} \leq n} \frac{\partial^{N-1} G}{\partial T_{i_{1}} \ldots \partial T_{i_{N-1}}}\left(f_{1}, \ldots, f_{n}\right) \frac{d f_{i_{1}}}{d T} \cdots \frac{d f_{i_{N-1}}}{d T}+\alpha\right),
$$

some $\alpha \in I_{N-2}$. As $\frac{d}{d T}\left(I_{N-2}\right) \subseteq I_{N-1}$ this equals, modulo $I_{N-1}$ :

$$
\begin{aligned}
\sum_{1 \leq i_{1}, \ldots, i_{N-1} \leq n}[ & \left(\sum_{j=1}^{n} \frac{\partial^{N} G}{\partial T_{i_{1}} \ldots \partial T_{i_{N-1}} \partial T_{j}}\left(f_{1}, \ldots, f_{n}\right) \frac{d f_{j}}{d T}\right) \frac{d f_{i_{1}}}{d T} \cdots \frac{d f_{i_{N-1}}}{d T} \\
& \left.+\frac{\partial^{N-1} G}{\partial T_{i_{1}} \ldots \partial T_{i_{N-1}}}\left(f_{1}, \ldots, f_{n}\right) \frac{d}{d T}(\ldots)\right] \\
\stackrel{i_{N}:=j}{=} & \sum_{1 \leq i_{1}, \ldots, i_{N} \leq n} \frac{\partial^{N} G}{\partial T_{i_{1}} \ldots \partial T_{i_{N}}}\left(f_{1}, \ldots, f_{n}\right) \frac{d f_{i_{1}}}{d T} \cdots \frac{d f_{i_{N}}}{d T},
\end{aligned}
$$

as claimed.

Proof of Theorem 2, Put

$$
R:=K_{0}\left(\operatorname{Rep}_{G_{k}} \mathbb{Q}_{l}\right), f_{i}(T):=\mu_{k}\left(\left[X_{i}\right]\right)=\sum_{\nu \geq 0} \operatorname{Gr}_{\nu}^{W}\left(X_{i}\right) T^{\nu} \in R[T],
$$

$a_{i}:=\operatorname{Gr}_{0}^{W}\left(X_{i}\right) \in \mathbb{Z} \subset R$ by assumption. Assume that we have $G \in \mathbb{Z}\left[T_{1}, \ldots, T_{n}\right]$ with $G\left(\mu_{k}\left(\left[X_{1}\right]\right), \ldots, \mu_{k}\left(\left[X_{n}\right]\right)\right)=0$, i.e.

$$
\tilde{G}(T):=G\left(f_{1}(T), \ldots, f_{n}(T)\right)=0 .
$$

We have to show that $G=0$ which follows from the Claim. For $N \geq 0$ and any $\underline{\alpha} \in \mathbb{N}_{0}^{n}$ with $|\underline{\alpha}| \leq N$ we have

$$
\frac{\partial^{|\underline{\alpha}|} G}{\partial T_{1}^{\alpha_{1}} \ldots \partial T_{n}^{\alpha_{n}}}\left(a_{1}, \ldots, a_{n}\right)=0
$$


which in turn will be established by induction on $N$. Note that $f_{i}(0)=a_{i}$, so from (9) putting $T=0$ we get $G\left(a_{1}, \ldots, a_{n}\right)=0$, i.e. the claim for $N=0$. Now assume that $N \geq 1$. By applying Proposition 4 to (9) and putting $T=0$ we obtain the following relation in $R$ :

$0=\frac{d^{N} \tilde{G}}{d T^{N}}(T=0)=\sum_{1 \leq i_{1}, \ldots, i_{N} \leq n} \frac{\partial^{N} G}{\partial T_{i_{1}} \ldots \partial T_{i_{N}}}\left(a_{1}, \ldots, a_{n}\right) \frac{d f_{i_{1}}}{d T}(0) \ldots \frac{d f_{i_{N}}}{d T}(0)+\alpha(0)$.

Here $\alpha \in I_{N-1}$, hence $\alpha(0)=0$ by induction hypothesis. As $\frac{d f_{i}}{d T}(0)=\operatorname{Gr}_{1}^{W}\left(X_{i}\right)$ we have the following relation in $R$ :

$$
0=\sum_{1 \leq i_{1}, \ldots, i_{N} \leq n} \frac{\partial^{N} G}{\partial T_{i_{1}} \ldots \partial T_{i_{N}}}\left(a_{1}, \ldots, a_{n}\right) \operatorname{Gr}_{1}^{W}\left(X_{i_{1}}\right) \ldots \operatorname{Gr}_{1}^{W}\left(X_{i_{N}}\right) .
$$

We collect terms in this expression: Consider

$$
\begin{aligned}
\pi: I:=\left\{\left(i_{1}, \ldots, i_{N}\right): 1 \leq i_{j} \leq n\right\} & \longrightarrow\left\{\underline{\alpha}=\left(\alpha_{1}, \ldots, \alpha_{n}\right) \in \mathbb{N}_{0}^{n}:|\underline{\alpha}|=N\right\} \\
\left(i_{1}, \ldots, i_{N}\right) & \longmapsto\left(\alpha_{k}:=\left|\left\{1 \leq j \leq N: i_{j}=k\right\}\right|\right)_{k=1, \ldots, n} .
\end{aligned}
$$

Given $\left(i_{1}, \ldots, i_{N}\right) \in I$ with $\pi\left(\left(i_{1}, \ldots, i_{N}\right)\right)=\left(\alpha_{1}, \ldots, \alpha_{n}\right)$ we have

$$
\frac{\partial^{N} G}{\partial T_{i_{1}} \ldots \partial T_{i_{N}}}=\frac{\partial^{N} G}{\partial T_{1}^{\alpha_{1}} \ldots \partial T_{n}^{\alpha_{n}}}
$$

and

$$
\operatorname{Gr}_{1}^{W}\left(X_{i_{1}}\right) \ldots \operatorname{Gr}_{1}^{W}\left(X_{i_{N}}\right)=\operatorname{Gr}_{1}^{W}\left(X_{1}\right)^{\alpha_{1}} \ldots \operatorname{Gr}_{1}^{W}\left(X_{n}\right)^{\alpha_{n}}
$$

So (10) may be written as

$$
\begin{gathered}
\sum_{\underline{\alpha}=\left(\alpha_{1} \ldots \alpha_{n}\right) \in \frac{\mathbb{N}_{0}^{n}}{|\underline{\alpha}|}=N}\left|\pi^{-1}(\underline{\alpha})\right| \frac{\partial^{N} G}{\partial T_{1}^{\alpha_{1}} \ldots \partial T_{n}^{\alpha_{n}}}\left(a_{1}, \ldots, a_{n}\right) \\
\cdot \operatorname{Gr}_{1}^{W}\left(X_{1}\right)^{\alpha_{1}} \ldots \operatorname{Gr}_{1}^{W}\left(X_{n}\right)^{\alpha_{n}}=0 .
\end{gathered}
$$

The $\operatorname{Gr}_{1}^{W}\left(X_{i}\right) \in R$ are algebraically independent by assumption and $\pi$ is surjective, hence $\left|\pi^{-1}(\underline{\alpha})\right| \neq 0$. So (11) implies

$$
\frac{\partial^{N} G}{\partial T_{1}^{\alpha_{1}} \ldots \partial T_{n}^{\alpha_{n}}}\left(a_{1}, \ldots, a_{n}\right)=0
$$

for all $\underline{\alpha} \in \mathbb{N}_{0}^{n}$ with $|\underline{\alpha}|=N$, concluding the proof of the Claim and of Theorem 2 .

2.3. Finite base field. In the situation of Section 2.1 we now assume in addition that the base field $k=\mathbb{F}_{q}$ is a finite field. We will use the fact that $G_{\mathbb{F}_{q}}$ is topologically cyclic, generated by the geometric Frobenius $F_{q} \in G_{\mathbb{F}_{q}}$, to rewrite the results of Sections 2.1 and 2.2 in terms of characteristic polynomials of Frobenius. We need to recall some facts about the "universal ring" from [DG], V, §5, no. 2.

For any commutative unitary ring $R$ the set $\Lambda(R):=1+t R[[t]]$ of formal power series with coefficients in $R$ and constant coefficient 1 is an abelian group under multiplication. The resulting functor $R \mapsto \Lambda(R)$ can be endowed with the structure of a functor in commutative unitary rings such that for all $R$ and $a, b \in R$

$$
(1-a t)(1-b t)=1-(a b) t \text { in } \Lambda(R)=1+t R[[t]] ;
$$


see loc. cit. Section 2.2. The multiplicative unit of $\Lambda(R)$ is $1-t$. The ring structure of $\Lambda(R)$ may cause confusion: The addition is the usual multiplication of power series, but the multiplication in $\Lambda(R)$ is a rather unusual operation on power series. We will denote addition and multiplication in $\Lambda(R)$ by the usual symbols but make clear that the composition is to be understood in $\Lambda(R)$. In this sense, the additive analogue of (12) is $(1-a t)+(1-b t)=1-(a+b) t+a b t^{2}$ in $\Lambda(R)$.

Let $R$ and $f \in \Lambda(R)$ be given. One defines $\partial_{\nu}(f) \in R$ for all $\nu \geq 1$ by the expansion $\operatorname{dlog}(f)=:-\sum_{\nu \geq 1} \partial_{\nu}(f) t^{\nu-1}$. Here, $\operatorname{dlog}(f)=\frac{d f / d t}{f}$ (usual quotient of power series) is the logarithmic derivative of $f$. If we denote by $\mathcal{O}$ the identity functor on the category of commutative unitary rings, then each $\partial_{\nu}: \Lambda \longrightarrow \mathcal{O}$ is a natural transformation (loc. cit. 2.4) and if $R$ is a $\mathbb{Q}$-algebra

$$
\Lambda(R) \stackrel{\sim}{\longrightarrow} R^{\mathbb{N}}, f \mapsto\left(\partial_{\nu}(f)\right)_{\nu \geq 1}
$$

is an isomorphism of rings in which $R^{\mathbb{N}}$ is the usual product of countably many copies of the ring $R$. For any $\nu \geq 1$ there is a natural transformation of ring-valued functors ("Frobenius") $F_{\nu}: \Lambda \longrightarrow \Lambda$ characterized by

$$
F_{\nu}(1-a t)=1-a^{\nu} t \text { in } \Lambda(R)=1+t R[[t]],
$$

for all $R$ and $a \in R$ and a natural transformation of functors in abelian groups ("Verschiebung") $V_{\nu}: \Lambda \longrightarrow \Lambda$ characterized by $V_{\nu}(1-a t)=1-a t^{\nu}$ in $\Lambda(R)$. In fact, we have

$$
V_{\nu}(f(t))=f\left(t^{\nu}\right) \text { for all } f(t) \in \Lambda(R)=1+t R[[t]] ;
$$

see loc. cit. 2.5 .

The rings $\Lambda(R)$ are convenient tools to keep track of characteristic polynomials.

Proposition 5. Let $k$ be a field and $V, W$ finite-dimensional $k$-vector spaces.

i) For $\phi \in \operatorname{End}_{k}(V)$ and $\psi \in \operatorname{End}_{k}(W)$ we have $\operatorname{det}(1-(\phi \oplus \psi) t \mid V \oplus W)=\operatorname{det}(1-\phi t \mid V)+\operatorname{det}(1-\psi t \mid W)$ and $\operatorname{det}(1-(\phi \otimes \psi) t \mid V \otimes W)=\operatorname{det}(1-\phi t \mid V) \operatorname{det}(1-\psi t \mid W)$ in $\Lambda(k)=1+t k[[t]]$. For any $\nu \geq 1$ : $\operatorname{det}\left(1-\phi^{\nu} t \mid V\right)=F_{\nu}(\operatorname{det}(1-\phi t \mid V))$ in $\Lambda(k)$ and $\partial_{\nu}(\operatorname{det}(1-\phi t \mid V))=\operatorname{tr}\left(\phi^{\nu} \mid V\right)$ in $k$.

ii) Let $G$ be a group and $N \subset G$ a normal subgroup such that $G / N$ is finite cyclic of order $\nu$ generated by the class of $\pi \in G$. If $V$ is a $k[N]$-module, finite-dimensional as a $k$-vector space, then we have

$$
\operatorname{det}\left(1-\pi t \mid \operatorname{Ind}_{N}^{G}(V)\right)=V_{\nu}\left(\operatorname{det}\left(1-\pi^{\nu} t \mid V\right)\right) \text { in } \Lambda(k)=1+t k[[t]] .
$$

In ii) we denoted by $\operatorname{Ind}_{N}^{G}(V):=k[G] \otimes_{k[N]} V$ the $k[G]$-module induced by $V$. Note that $\pi^{\nu} \in N$, hence the right-hand side of (16) is meaningful.

Proof. Part i) follows from equations (12) and (14) and [H], Appendix C, Lemma 4.1. Using (15), part ii) is equivalent to $\operatorname{det}\left(1-\pi t \mid \operatorname{Ind}_{N}^{G}(V)\right)=\operatorname{det}\left(1-\pi^{\nu} t^{\nu} \mid V\right)$. This has to be checked by an explicit computation which we omit.

For a ring $R$ we define a subset $\mathcal{R}(R) \subset \Lambda(R)$ by

$$
\mathcal{R}(R):=\{f-g \mid f, g \in 1+t R[t]\} \subset \Lambda(R)=1+t R[[t]] .
$$

It is clear that $\mathcal{R} \subset \Lambda$ is a subfunctor in abelian groups. One should think of the elements of $\mathcal{R}(R)$ as the the power series expansions of certain rational functions, namely the quotients of polynomials with constant coefficient 1 . 
Proposition 6. $\mathcal{R} \subset \Lambda$ is a subfunctor in rings.

Proof. First, $1_{\Lambda(R)}=1-t \in \mathcal{R}(R) \subset \Lambda(R)=1+t R[[t]]$. Now it suffices to check that for $f, g \in 1+t R[t]$ we have $f g \in \mathcal{R}(R) \subset \Lambda(R)$. Indeed, $f g$ is again a polynomial according to $[\mathrm{DG}]$, p. 632 , formula $(* *)$.

Using Proposition 5 one sees that there is a unique ring homomorphism

$$
\chi_{q}: K_{0}\left(\operatorname{Rep}_{G_{\mathbb{F}_{q}}} \mathbb{Q}_{l}\right) \hookrightarrow \Lambda\left(\mathbb{Q}_{l}\right)
$$

such that

$$
\chi_{q}([V])=\operatorname{det}\left(1-F_{q} t \mid V\right) \in \Lambda\left(\mathbb{Q}_{l}\right)=1+t \mathbb{Q}_{l}[[t]] \text { for } V \in \operatorname{Rep}_{G_{\mathbb{F}_{q}}} \mathbb{Q}_{l}
$$

and $\chi_{q}$ is injective as follows from Algèbre, $§ 12,1$, Proposition 3.

Composing (6) with $\chi_{q}$ we obtain a motivic measure

$$
\mu_{q}: K_{0}\left(\operatorname{Var}_{\mathbb{F}_{q}}\right) \longrightarrow \Lambda\left(\mathbb{Q}_{l}\right)[T]
$$

given explicitly by

$$
\mu_{q}([X])=\sum_{i \geq 0}\left(\sum_{j}(-1)^{j} \operatorname{det}\left(1-F_{q} t \mid \operatorname{Gr}_{i}^{W}\left(H_{c}^{j}(\bar{X})\right)\right)\right) T^{i} .
$$

Proposition 7. The image of (17) is contained in $\mathcal{R}(\mathbb{Q})[T] \subset \Lambda\left(\mathbb{Q}_{l}\right)[T]$.

Proof. Let $X / \mathbb{F}_{q}$ be separated and of finite type. The cohomological formula for the zeta function $Z_{X / \mathbb{F}_{q}}(t)$ of $X / \mathbb{F}_{q}\left(\left[\mathrm{Mi2}\right.\right.$, , Theorem 13.1) gives in $\Lambda\left(\mathbb{Q}_{l}\right)=1+t \mathbb{Q}_{l}[[t]]$ :

$$
\begin{aligned}
& Z_{X / \mathbb{F}_{q}}(t)=\sum_{j \geq 0}(-1)^{j+1} \operatorname{det}\left(1-F_{q} t \mid H_{c}^{j}(\bar{X})\right) \\
& =\sum_{j \geq 0}(-1)^{j+1}\left(\sum_{i} \operatorname{det}\left(1-F_{q} t \mid \operatorname{Gr}_{i}^{W}\left(H_{c}^{j}(\bar{X})\right)\right)\right) \\
& =\sum_{i}\left(\sum_{j \geq 0}(-1)^{j+1} \operatorname{det}\left(1-F_{q} t \mid \operatorname{Gr}_{i}^{W}\left(H_{c}^{j}(\bar{X})\right)\right)\right) .
\end{aligned}
$$

In this expression, every summand is in $\mathbb{Q}_{l}(t)$, and we have that $Z_{X / \mathbb{F}_{q}}(t) \in \mathbb{Q}[[t]]$ from the very definition of $Z_{X / \mathbb{F}_{q}}(t)$. For weight reasons there can be no cancellation among different summands (recall that the sums are actually products of power series), hence every summand lies in $\mathbb{Q}_{l}(t) \cap \mathbb{Q}[[t]] \subset \mathbb{Q}(t)$. As every summand is in addition a quotient of polynomials with constant coefficient 1 , every summand lies in fact in $\mathcal{R}(\mathbb{Q})$ and comparison with (18) concludes the proof.

The relevance of having a motivic measure taking values in $\mathcal{R}(\mathbb{Q})[T]$ rather than in $\Lambda\left(\mathbb{Q}_{l}\right)[T]$ will become clear in Section 3.2. Finally we record some formulae relating the various operations on $\Lambda$ with the motivic measure $\mu_{q}$.

\section{Proposition 8.}

i) If $X / \mathbb{F}_{q}$ is separated and of finite type, then, for all $\nu \geq 1$, $\partial_{\nu}\left(\mu_{q}([X])\right)=\sum_{i \geq 0}\left(\sum_{j}(-1)^{j} \operatorname{tr}\left(F_{q}^{\nu} \mid \operatorname{Gr}_{i}^{W}\left(H_{c}^{j}(\bar{X})\right)\right)\right) T^{i}$ in $\mathbb{Q}[T]$. 
ii) For $\mathbb{F}_{q} \subset \mathbb{F}_{q^{\nu}} \subset \overline{\mathbb{F}_{q}}$ the finite extension of degree $\nu \geq 1$ we have a commutative diagram

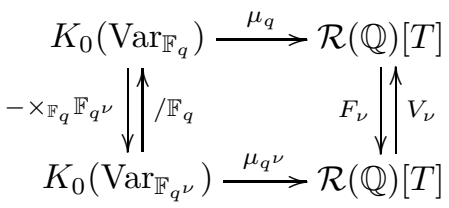

In i) $\partial_{\nu}$ denotes the restriction of $\partial_{\nu}: \Lambda(\mathbb{Q}) \longrightarrow \mathbb{Q}$ to $\mathcal{R}(\mathbb{Q}) \subset \Lambda(\mathbb{Q})$ extended to $\partial_{\nu}: \mathcal{R}(\mathbb{Q})[T] \longrightarrow \mathbb{Q}[T]$ by demanding that $\partial_{\nu}(T)=T$, and in ii) $F_{\nu}$ and $V_{\nu}$ denote the maps which on $\mathcal{R}(\mathbb{Q})$ are the restrictions of $F_{\nu}, V_{\nu}: \Lambda(\mathbb{Q}) \longrightarrow \Lambda(\mathbb{Q})$ and with $F_{\nu}(T)=T$ and $V_{\nu}(T)=T$.

Proof. Part i) follows from (18) and Proposition (5). Part ii) follows from (8) and Proposition 5) and ii).

\section{Algebraic independence}

3.1. Virtual continuous representations. In view of Corollary 3 we are led to consider the following problem:

Given a topological group $T$ and a finite extension $K / \mathbb{Q}_{l}$ denote by $\operatorname{Rep}_{T} K$ the category of finite-dimensional continuous representations of $T$ over $K$. Given finitely many $V_{i} \in \operatorname{Rep}_{T} K$, when are their classes $\left[V_{i}\right] \in K_{0}\left(\operatorname{Rep}_{T} K\right)$ algebraically independent?

In this subsection we will use a Tannakian argument to reduce this question to a problem about algebraic independence of representations of a (possibly nonconnected) reductive algebraic group and in a special case of a diagonalizable group. For this latter case we establish a rather explicit Jacobi criterion (Lemma 10). The subtleties arising from the possible non-connectedness of the reductive group above will be illustrated by an example (Theorem 11) and motivate the following definition.

Definition 9. Representations $V_{i} \in \operatorname{Rep}_{T} K$ are geometrically algebraically independent if for all open subgroups of finite index $T^{\prime} \subset T$ the classes of the restrictions $\left[\operatorname{Res}_{T^{\prime}}^{T}\left(V_{i}\right)\right] \in K_{0}\left(\operatorname{Rep}_{T^{\prime}} K\right)$ are algebraically independent.

We now give a series of reduction steps to obtain workable criteria for the $\left[V_{i}\right] \in$ $K_{0}\left(\operatorname{Rep}_{T} K\right)$ to be algebraically (in)dependent. The reader is invited to glance at a simple application, Theorem [11, first.

Denoting by $V_{i}^{s s}$ the semi-simplification of $V_{i}$ we have

$$
\left[V_{i}\right]=\left[V_{i}^{s s}\right] \text { in } K_{0}\left(\operatorname{Rep}_{T} K\right) \text {, }
$$

and we can assume the $V_{i}$ to be semi-simple themselves. In the sequel we will repeatedly use the obvious fact that elements $x_{i} \in R \subset S$ of a subring $R$ of $S$ are algebraically independent in $R$ if and only if they are so in $S$. We now use a Tannakian argument to find an accessible subring of $K_{0}\left(\operatorname{Rep}_{T} K\right)$ containing all the $\left[V_{i}\right]$. The reference for Tannakian categories is [D2]. With the obvious notion of tensor product, dual and using the forgetful functor $\operatorname{Rep}_{T} K \longrightarrow$ Vect $_{K}$ to the category of finite-dimensional $K$-vector spaces as a fibre functor, the category $\operatorname{Rep}_{T} K$ is a neutral Tannakian category over $K$. We put $V:=\bigoplus_{i=1}^{n} V_{i}$ and consider

$$
i: \mathcal{C}:=\langle V\rangle^{\otimes} \hookrightarrow \operatorname{Rep}_{T} K,
$$


the Tannakian subcategory generated by $V$, i.e. the smallest strictly full subcategory of $\operatorname{Rep}_{T} K$ containing $V$ and stable under the formation of finite direct sums, tensor products, duals, subobjects and quotients. As $i$ is an exact tensor functor it induces a ring homomorphism

$$
K_{0}(\mathcal{C}) \hookrightarrow K_{0}\left(\operatorname{Rep}_{T} K\right)
$$

which is injective: one checks that $i$ preserves simple objects and uses the fact that the $K_{0}$ of an abelian category with all objects of finite length is $\mathbb{Z}$-free on the isomorphism classes of its simple objects Q,$\S 5$, Corollary 1. By construction we have $\left[V_{i}\right] \in K_{0}(\mathcal{C})$, and our initial problem is reduced to considering the algebraic (in)dependence of the $\left[V_{i}\right]$ as elements of $K_{0}(\mathcal{C})$. Let

$$
\phi: T \longrightarrow \mathrm{Gl}(V)(K)
$$

be the continuous homomorphism giving the action of $T$ on $V$. Here Gl $(V)$ denotes the algebraic group and $\mathrm{Gl}(V)(K)$ its group of $K$-points endowed with the topology inherited from the one of $K$. The Zariski closure of the image of $\phi$, say

$$
G:=\overline{\operatorname{im}(\phi)} \subset \operatorname{Gl}(V),
$$

is an affine algebraic group over $K([\overline{B o}$, Proposition 1.3, b) $)$ and can be identified with the Tannaka group of $\mathcal{C}$ as follows ([S4, 1.3). From $\phi$ we have a continuous homomorphism $\tilde{\phi}: T \longrightarrow G(K)$, and the restriction of the fibre functor $\mathcal{C} \longrightarrow$ Vect $_{K}$ induces an equivalence of Tannakian categories

$$
\mathcal{C} \stackrel{\sim}{\longrightarrow} \underline{\operatorname{Rep}}_{K} G,
$$

where $\operatorname{Rep}_{K} G$ denotes the category of finite-dimensional representations of the algebraic group $G$ over $K$. A quasi-inverse of (24) is given by

$$
\underline{\operatorname{Rep}}_{K} G \stackrel{\sim}{\longrightarrow} \mathcal{C},(G \stackrel{\psi}{\longrightarrow} \mathrm{Gl}(W)) \mapsto \psi(K) \circ \tilde{\phi} .
$$

As $\mathcal{C}$ is semi-simple by (20), then DMOS, Remark 2.28, implies that $G$ is (not necessarily connected) reductive. The isomorphism $K_{0}(\mathcal{C}) \simeq K_{0}\left(\underline{\operatorname{Rep}}_{K} G\right)$ induced by (24) reduces our initial problem to studying the algebraic (in)dependence of given representations of a reductive group. We next show that we may allow finite extensions of the coefficients $K$ of these representations. For $K \subset L$ a finite extension, the exact tensor functor

$$
\underline{\operatorname{Rep}}_{K} G \longrightarrow \underline{\operatorname{Rep}}_{L} G \otimes L, V \mapsto V \otimes_{K} L
$$

induces a ring homomorphism

$$
K_{0}\left(\underline{\operatorname{Rep}}_{K} G\right) \hookrightarrow K_{0}\left(\underline{\operatorname{Rep}}_{L} G \otimes L\right)
$$

which is injective; cf. Mi1, Lemma 3.14, for a similar argument. This allows us to replace $G$ by $G \otimes L$ and to assume that the connected component of identity $G^{0} \subset G$ is a connected split reductive group and that the finite étale group $G / G^{0}$ is in fact constant. Extending $K$ further to contain a splitting field of $G / G^{0}$, the ring $K_{0}\left(\underline{\operatorname{Rep}}_{K} G / G^{0}\right)$ is a familiar object from the representation theory of finite (abstract) groups $\underline{\mathrm{S} 3}$, Chapter II. Also $K_{0}\left(\underline{\operatorname{Rep}}_{K} G^{0}\right)$ can be described quite precisely (cf. below) but without imposing further, rather restrictive, conditions (e.g. $\left.G \simeq G^{0} \times G / G^{0}\right)$ I cannot say much about the structure of $K_{0}\left(\underline{\operatorname{Rep}}_{K} G\right)$. We will describe two special situations in which the above line of thought may be continued. 
So $K / \mathbb{Q}_{l}$ is a finite extension, $G$ is a split reductive group over $K$ and we need to study algebraic (in)dependence in the ring $K_{0}\left(\underline{\operatorname{Rep}}_{K} G\right)$. We assume in addition that $G$ is connected and fix a $K$-split maximal torus $S \subset G$. Then restriction induces an injective ring homomorphism

$$
K_{0}\left(\underline{\operatorname{Rep}}_{K} G\right) \hookrightarrow K_{0}\left(\underline{\operatorname{Rep}}_{K} S\right)
$$

[S1, 3.4, and the structure of $K_{0}\left(\underline{\operatorname{Rep}}_{K} S\right)$ is particularly simple; cf. (28) for the more general case of a diagonalizable group. It then becomes important to determine the character group of $S$ which in the present case (the topological group $T$ being arbitrary) we do not know how to do. The most satisfactory solution of our initial problem can be obtained in the case that $T=\langle F\rangle$ is topologically cyclic. Though very special, this case will be of interest for us because it covers the case of the Galois group of a finite field. So let

$$
\phi: T=\langle F\rangle \longrightarrow \mathrm{Gl}(V)(K) \simeq \mathrm{Gl}_{m}(K)
$$

be as in (22) (so $m=\sum_{i=1}^{n} \operatorname{dim}{ }_{K} V_{i}$ ). By our preliminary reductions (20) and (25) and conjugating $\phi(F)$ suitably we can assume that

$$
\phi(F)=\operatorname{diag}\left(\alpha_{1}, \ldots, \alpha_{m}\right) \in \mathbb{D}_{m}(K)
$$

for some $\alpha_{i} \in K^{*}$, where $\mathbb{D}_{m} \subset \mathrm{Gl}_{m}$ is the torus of diagonal matrices. Then $G$ as in (23) is the smallest algebraic subgroup $G \subset \mathrm{Gl}_{m}$ defined over $K$ such that $\phi(F) \in G(K)$, and $D:=G \subset \mathbb{D}_{m}$ is a closed subgroup, hence diagonalizable by [Bo, Proposition 8.4. The character group $X(D)$ of $D$ can be identified with the subgroup of $K^{*}$ generated by the eigenvalues of $\phi(F)$ as follows from [Bo], 8.2, Corollary:

$$
X(D) \simeq\left\langle\alpha_{1}, \ldots, \alpha_{m}\right\rangle \subset K^{*} .
$$

We recall the structure of $K_{0}\left(\underline{\operatorname{Rep}}_{K} D\right)$ from [S1], 3.4. Giving $V \in \underline{\operatorname{Rep}}_{K} D$ is equivalent to giving a "grading of type $X(D)$ " of the finite-dimensional $K$-vector space $V$, i.e. $V=\bigoplus_{\chi \in X(D)} V_{\chi}$, where $V_{\chi} \subset V$ is the $\chi$-eigenspace and

$$
\operatorname{ch}: K_{0}\left(\underline{\operatorname{Rep}}_{K} D\right) \stackrel{\sim}{\longrightarrow} \mathbb{Z}[X(D)],[V] \mapsto \sum_{\chi} \operatorname{dim}_{K}\left(V_{\chi}\right) e^{\chi}
$$

is a ring isomorphism. Here $\mathbb{Z}[X(D)]$ is the group algebra over $X(D)$, i.e. $\mathbb{Z}[X(D)]$ is $\mathbb{Z}$-free on the set $\left\{e^{\chi}: \chi \in X(D)\right\}$ and $e^{\chi} e^{\chi^{\prime}}=e^{\chi+\chi^{\prime}}$. Decomposing the finitely generated abelian group $X(D)$ as

$$
X(D)=X(D)^{t o r} \bigoplus_{i=1}^{d} \mathbb{Z} e_{i},
$$

where $X(D)^{\text {tor }} \subset X(D)$ is the torsion subgroup and the $e_{i} \in X(D)$ are suitably chosen, the natural map

$$
\mathbb{Z}\left[X(D)^{\text {tor }}\right] \otimes_{\mathbb{Z}} \mathbb{Z}\left[\bigoplus_{i=1}^{d} \mathbb{Z} e_{i}\right] \stackrel{\sim}{\longrightarrow} \mathbb{Z}[X(D)], e^{t} \otimes e^{f} \mapsto e^{t+f}
$$

is an isomorphism and

$$
\mathbb{Z}\left[\bigoplus_{i=1}^{d} \mathbb{Z} e_{i}\right] \stackrel{\sim}{\longrightarrow} \mathbb{Z}\left[T_{1}, \ldots, T_{d}, T_{1}^{-1}, \ldots, T_{d}^{-1}\right], e^{\sum a_{i} e_{i}} \mapsto T_{1}^{a_{1}} \ldots T_{d}^{a_{d}}
$$


is an isomorphism to the ring of Laurant polynomials over $\mathbb{Z}$ in

$$
d=\operatorname{rk}(X(D))=\operatorname{dim}(D)
$$

variables. Since $X(D)^{\text {tor }}$ is finite, $A:=\mathbb{Z}\left[X(D)^{\text {tor }}\right]$ is a finite flat $\mathbb{Z}$-algebra and summing up we have

$$
K_{0}\left(\underline{\operatorname{Rep}}_{K} D\right) \simeq A\left[T_{1}, \ldots, T_{d}, T_{1}^{-1}, \ldots, T_{d}^{-1}\right] .
$$

Note that, if $X(D)$ is as in (27), then $X(D)^{\text {tor }} \subset K^{*}$ is a finite subgroup, hence cyclic of order $N$, say. Then we have

$$
A=\mathbb{Z}[\mathbb{Z} / N] \simeq \mathbb{Z}[S] /\left(S^{N}-1\right) .
$$

So our initial problem is finally reduced to deciding whether the

$$
f_{i}:=\operatorname{ch}\left(\left[V_{i}\right]\right) \in A\left[T_{1}, \ldots, T_{d}, T_{1}^{-1}, \ldots, T_{d}^{-1}\right],
$$

i.e. the characters of the given representations $V_{i}$, are algebraically independent. For this problem one can prove a Jacobian criterion as follows.

Let $A$ be a finite flat $\mathbb{Z}$-algebra. We will prove a criterion for given

$$
f_{1}, \ldots, f_{n} \in A\left[T_{1}, \ldots, T_{m}, T_{1}^{-1}, \ldots, T_{m}^{-1}\right]
$$

to be algebraically independent in terms of the Jacobian

$$
J:=\left(\frac{\partial f_{i}}{\partial T_{j}}\right)_{1 \leq i \leq n, 1 \leq j \leq m}
$$

which is an $n$ by $m$ matrix with entries from $A\left[T_{1}, \ldots, T_{m}, T_{1}^{-1}, \ldots, T_{m}^{-1}\right]$.

For a homomorphism $\phi: A \rightarrow \overline{\mathbb{Q}}$ we denote by

$$
\begin{aligned}
\phi \otimes 1: A\left[\underline{T}, \underline{T}^{-1}\right]: & :=A\left[T_{1}, \ldots, T_{m}, T_{1}^{-1}, \ldots, T_{m}^{-1}\right]=A \otimes \mathbb{Z}\left[\underline{T}, \underline{T}^{-1}\right] \\
\rightarrow \overline{\mathbb{Q}}\left[\underline{T}, \underline{T}^{-1}\right]:=\overline{\mathbb{Q}}\left[T_{1}, \ldots, T_{m}, T_{1}^{-1}, \ldots, T_{m}^{-1}\right] & =\overline{\mathbb{Q}} \otimes \mathbb{Z}\left[\underline{T}, \underline{T}^{-1}\right]
\end{aligned}
$$

the base change of $\phi$. Then $(\phi \otimes 1)(J)$ is a matrix with coefficients in

$$
\overline{\mathbb{Q}}\left[\underline{T}, \underline{T}^{-1}\right] \subset Q:=\overline{\mathbb{Q}}\left(T_{1}, \ldots, T_{m}\right),
$$

where $Q$ is the field of rational functions in $m$ variables over $\overline{\mathbb{Q}}$. For a homomorphism $\psi: \overline{\mathbb{Q}}\left[\underline{T}, \underline{T}^{-1}\right] \longrightarrow K$ with $K$ some field, $(\psi \circ(\phi \otimes 1))(J)$ is a matrix with coefficients in $K$. Finally, by $\operatorname{rk}_{L}(M)$ we will denote the rank of a matrix $M$ having coefficients in the field $L$. The Jacobi criterion for algebraic independence mentioned above is the following.

Lemma 10. Keeping the above notations we have:

1) The following are equivalent:

i) The $f_{i} \in A\left[\underline{T}, \underline{T}^{-1}\right]$ are algebraically dependent.

ii) For all $\phi: A \rightarrow \overline{\mathbb{Q}}$ we have

$$
\operatorname{rk}_{Q}((\phi \otimes 1)(J))<n .
$$

2) The following are equivalent:

i) The $f_{i} \in A\left[\underline{T}, \underline{T}^{-1}\right]$ are algebraically independent.

ii) There are homomorphisms $\phi: A \rightarrow \overline{\mathbb{Q}}$ and $\psi: \overline{\mathbb{Q}}\left[\underline{T}, \underline{T}^{-1}\right] \rightarrow K$ (K some field) such that

$$
\operatorname{rk}_{K}(\psi \circ(\phi \otimes 1)(J))=n .
$$


The proof of Lemma 10 consists in a reduction, which we will omit, to the case when $A$ is a field of characteristic zero. In this case the Lemma is $[\mathrm{Bo}$, Chapter AG, Theorem 17.3. We conclude this subsection by giving two applications.

Theorem 11. Let $k$ be a finite field and let $E_{1}, E_{2} / k$ be non-isogeneous ordinary elliptic curves. Let $E_{2}^{\prime} / k$ be the quadratic twist of $E_{2}$, and consider the abelian surfaces $A_{1}:=E_{1} \times E_{2}$ and $A_{2}:=E_{1} \times E_{2}^{\prime}$ over $k$ and the associated Galois representations $V_{i}:=H_{c}^{1}\left(\overline{A_{i}}\right) \in \operatorname{Rep}_{G_{k}} \mathbb{Q}_{l}$. Let $k \subset L$ be the unique quadratic extension of $k$ inside $\bar{k}$. Then:

i) $\operatorname{Res}_{G_{L}}^{G_{k}}\left(V_{1}\right) \simeq \operatorname{Res}_{G_{L}}^{G_{k}}\left(V_{2}\right)$.

ii) The classes $\left[V_{1}\right]$ and $\left[V_{2}\right]$ are algebraically independent in $K_{0}\left(\operatorname{Rep}_{G_{k}} \mathbb{Q}_{l}\right)$.

In particular, the classes $\left[A_{1}\right]$ and $\left[A_{2}\right]$ are algebraically independent in $K_{0}\left(\operatorname{Var}_{k}\right)$.

Remark. The representations $V_{1}$ and $V_{2}$ are algebraically independent but not geometrically algebraically independent. In fact, after restriction to the subgroup of index two of $G_{k}$ their classes become algebraically dependent and in fact equal. We also see that $x:=\left[A_{1}\right]-\left[A_{2}\right] \in K_{0}\left(\operatorname{Var}_{k}\right)$ generates a polynomial ring over $\mathbb{Z}$ inside $K_{0}\left(\operatorname{Var}_{k}\right)$ even though $x$ lies in the kernel of the base change homomorphism $K_{0}\left(\operatorname{Var}_{k}\right) \longrightarrow K_{0}\left(\operatorname{Var}_{L}\right)$. This shows that $K_{0}\left(\operatorname{Var}_{k}\right)$ encodes fine arithmetic invariants of varieties over $k$.

Proof. Part i) is clear because $A_{1} \times_{k} L \simeq A_{2} \times_{k} L$, and the last assertion follows from part ii) and Corollary 3 , We prove ii): Fix $\pi_{i} \in \overline{\mathbb{Q}}^{*}$ a Weil number attached to $E_{i} / k$ and denote by $\bar{\pi}_{i}$ the conjugate of $\pi_{i}$. Note that $\mathbb{Q}\left(\pi_{1}\right)$ and $\mathbb{Q}\left(\pi_{2}\right)$ are distinct imaginary quadratic fields, hence

$$
\mathbb{Q}\left(\pi_{1}\right) \cap \mathbb{Q}\left(\pi_{2}\right)=\mathbb{Q} .
$$

The eigenvalues of the geometric Frobenius $F_{k}$ acting on the $H_{c}^{1}$ of $E_{1}, E_{2}$ and $E_{2}^{\prime}$ are (respectively) $\left\{\pi_{1}, \bar{\pi}_{1}\right\},\left\{\pi_{2}, \bar{\pi}_{2}\right\}$ and $\left\{-\pi_{2},-\bar{\pi}_{2}\right\}$. Fixing a finite extension $\mathbb{Q}_{l} \subset K$ containing $\pi_{1}$ and $\pi_{2}$ we get in the notation of (26):

$$
\phi\left(F_{k}\right)=\operatorname{diag}\left(\pi_{1}, \bar{\pi}_{1}, \pi_{2}, \bar{\pi}_{2}, \pi_{1}, \bar{\pi}_{1},-\pi_{2},-\bar{\pi}_{2}\right) \in \mathbb{D}_{8}(K)
$$

and

$$
X(D) \simeq\left\langle\pi_{1}, \bar{\pi}_{1}, \pi_{2}, \bar{\pi}_{2},-1\right\rangle \subset K^{*} .
$$

Using (31) and the fact that $E_{1}$ and $E_{2}$ are ordinary, one checks that the structure of $X(D)$ is as follows:

$$
X(D)=\{ \pm 1\} \oplus \pi_{1}^{\mathbb{Z}} \oplus\left(\bar{\pi}_{1}\right)^{\mathbb{Z}} \oplus \pi_{2}^{\mathbb{Z}} .
$$

From (32) we get as in (29) together with (30)

$$
K_{0}\left(\underline{\operatorname{Rep}}_{K} D\right) \simeq\left(\mathbb{Z}[S] /\left(S^{2}-1\right)\right)\left[T_{1}, T_{1}^{-1}, \ldots, T_{3}, T_{3}^{-1}\right]
$$

and compute the characters $f_{i}:=\operatorname{ch}\left(\left[V_{i}\right]\right)$ as $f_{1}=e^{\pi_{1}}+e^{\bar{\pi}_{1}}+e^{\pi_{2}}+e^{\bar{\pi}_{2}}$ and $f_{2}=e^{\pi_{1}}+e^{\bar{\pi}_{1}}+e^{-\pi_{2}}+e^{-\bar{\pi}_{2}}$. We have $\bar{\pi}_{2}=\pi_{1} \bar{\pi}_{1} \pi_{2}^{-1}$ and using this, (32) and (33) we get (denoting abusively the images of $\left[V_{i}\right]$ under (33) by $f_{i}$ again)

$$
\begin{aligned}
& f_{1}=T_{1}+T_{2}+T_{3}+T_{1} T_{2} T_{3}^{-1} \text { and } \\
& f_{2}=T_{1}+T_{2}+S T_{3}+S T_{1} T_{2} T_{3}^{-1} .
\end{aligned}
$$


To show that $f_{1}$ and $f_{2}$ are algebraically independent we compute the Jacobian of $f_{1}$ and $f_{2}$

$$
J=\left(\begin{array}{ccc}
1+T_{2} T_{3}^{-1} & 1+T_{1} T_{3}^{-1} & 1-T_{1} T_{2} T_{3}^{-2} \\
1+S T_{2} T_{3}^{-1} & 1+S T_{1} T_{3}^{-1} & S-S T_{1} T_{2} T_{3}^{-2}
\end{array}\right)
$$

which we specialize by putting $S=-1, T_{1}=-1, T_{2}=T_{3}=1$ to obtain

$$
\left(\begin{array}{ccc}
2 & 0 & 2 \\
0 & 2 & -2
\end{array}\right)
$$

which has rank two, hence $f_{1}$ and $f_{2}$ are algebraically independent by Lemma 10, as was to be shown.

Our next application of the methods developed so far is the following.

Theorem 12. Let $k$ be a finite field. Then there is a sequence of proper, smooth and geometrically connected curves $X_{i} / k(i \geq 1)$ such that the classes $\left[X_{i}\right] \in K_{0}\left(\operatorname{Var}_{k}\right)$ are algebraically independent.

Theorem 13. Let $k$ be a number field and $\left\{E_{i}\right\}_{i \in I}$ a set of elliptic curves over $k$ such that the $E_{i}$ are pairwise non-isogeneous and satisfy $\operatorname{End}_{\bar{k}}\left(E_{i}\right)=\mathbb{Z}$. Then the classes $\left[E_{i}\right] \in K_{0}\left(\operatorname{Var}_{k}\right)$ are algebraically independent.

Note that over any number field there are infinite sets of elliptic curves satisfying the assumptions of Theorem 13 .

To prove these results we will need the following sufficient criterion for geometric algebraic independence in terms of Frobenius eigenvalues.

Lemma 14. Let $\hat{\mathbb{Z}}$ denote the pro-finite completion of $\mathbb{Z}$ and let $K / \mathbb{Q}_{l}$ be a finite extension and let $V_{1}, \ldots, V_{n} \in \operatorname{Rep}_{\hat{\mathbb{Z}}} K$ be given. For $1 \leq i \leq n$ put $m_{i}:=\operatorname{dim}_{K} V_{i}$ and let

$$
\left\{\lambda_{i, j}\right\}_{1 \leq j \leq m_{i}} \subset \bar{K}^{*}
$$

be the eigenvalues of $F:=1$ acting on $V_{i}$. For any $1 \leq k \leq n$ consider the finitedimensional $\mathbb{Q}$-vector space

$$
A_{k}:=\left\langle\lambda_{i, j}\right\rangle_{1 \leq i \leq k, 1 \leq j \leq m_{i}} \otimes_{\mathbb{Z}} \mathbb{Q}
$$

and set $A_{0}:=0$. If

$$
\operatorname{dim}_{\mathbb{Q}} A_{k}>\operatorname{dim}_{\mathbb{Q}} A_{k-1} \text { for } k=1, \ldots, n,
$$

then the $V_{1}, \ldots, V_{n} \in \operatorname{Rep}_{\hat{\mathbb{Z}}} K$ are geometrically algebraically independent.

For the application of this lemma in the proof of Theorem 13 we make its assumptions more explicit in the case when the $V_{i}$ are (dual to) the Tate-modules of elliptic curves.

Lemma 15. Let $\mathbb{F}_{q}$ be a finite field and identify $G_{\mathbb{F}_{q}}=\hat{\mathbb{Z}}$ using the geometric Frobenius. Let $E_{1}, \ldots, E_{n} / \mathbb{F}_{q}$ be elliptic curves, assume that $E_{1}$ is ordinary, put $V_{i}:=H_{c}^{1}\left(\overline{E_{i}}\right) \in \operatorname{Rep}_{G_{\mathbb{E}_{q}}}\left(\mathbb{Q}_{l}\right)$ and define the $A_{i}$ as in Lemma 14, Then the assumptions of this Lemma are satisfied if and only if $\operatorname{dim}_{\mathbb{Q}} A_{n}=n+1$.

Proof. Since $E_{1}$ is ordinary we have $\operatorname{dim}_{\mathbb{Q}} A_{1}=2$. For $k=2, \ldots, n$ we have $\operatorname{dim}_{\mathbb{Q}} A_{k} \leq \operatorname{dim}_{\mathbb{Q}} A_{k-1}+1$ because $\lambda_{k, 1} \lambda_{k, 2}=q \in A_{k-1}$. The assertion is obvious now. 
Proof of Lemma 14. In the notation of (26) we have

$$
\phi(F)=\operatorname{diag}\left(\lambda_{1,1}, \ldots, \lambda_{1, m_{1}}, \ldots, \lambda_{n, 1}, \ldots, \lambda_{n, m_{n}}\right) \in \mathbb{D}_{\sum_{i=1}^{n} m_{i}}(K) .
$$

As a first step we will prove that the characters $f_{i}:=\operatorname{ch}\left(\left[V_{i}\right]\right) \in K_{0}\left(\underline{\operatorname{Rep}}_{K} D\right)$ are algebraically independent. Here $D \subset \mathbb{D}_{\sum_{i=1}^{n} m_{i}}$ is the smallest algebraic subgroup with $\phi(F) \in D(K)$. After suitably enlarging $K$ (legitimate by (25)) we identify (as in (27)) the character group $X(D)$ of $D$,

$$
X(D) \simeq\left\langle\lambda_{i, j}\right\rangle_{1 \leq i \leq n, 1 \leq j \leq m_{i}} \subset K^{*},
$$

and choose a decomposition

$$
X(D)=\langle\zeta\rangle \bigoplus_{i=1}^{N} \pi_{i}^{\mathbb{Z}}
$$

with $\zeta \in K^{*}$ a root of unity of order $M$, say, and $\pi_{1}, \ldots, \pi_{N} \in K^{*}$ a $\mathbb{Z}$-basis of the free part of $X(D)$. We order the $\pi_{i}$ 's such that their images $\pi_{i} \otimes 1$ in $X(D) \otimes \mathbb{Q}=A_{n}$ satisfy the following: For all $1 \leq k \leq n, \pi_{1} \otimes 1, \ldots, \pi_{d_{k}} \otimes 1$ is a basis of $A_{k}$. Here $d_{k}:=\operatorname{dim}_{\mathbb{Q}} A_{k}$ and (35) gives

$$
d_{0}=0<d_{1}<\ldots<d_{n}=N .
$$

Note that

$$
\begin{aligned}
& \left.N=\operatorname{dim}(D)=\operatorname{dim}_{\mathbb{Q}}(X(D) \otimes \mathbb{Q})\right) \\
& \stackrel{\text { 366) }}{=} \operatorname{dim} \mathbb{Q}\left(\left\langle\lambda_{i, j}\right\rangle_{1 \leq i \leq n, 1 \leq j \leq m_{i}} \otimes \mathbb{Q}\right) \\
& \stackrel{\text { 344) }}{=} \operatorname{dim}{ }_{\mathbb{Q}}\left(A_{n}\right) \stackrel{\text { (35) }}{\geq} n .
\end{aligned}
$$

For any $1 \leq i \leq n$ and $1 \leq j \leq m_{i}$ we decompose, according to (37),

$$
\lambda_{i, j}=\zeta^{a_{i, j}} \pi_{1}^{a_{1, i, j}} \ldots \pi_{N}^{a_{N, i, j}}
$$

for suitable $a_{i, j}, a_{k, i, j} \in \mathbb{Z}$. Using (29)

$$
K_{0}\left(\underline{\operatorname{Rep}}_{K} D\right) \simeq A\left[T_{1}, T_{1}^{-1}, \ldots, T_{N}, T_{N}^{-1}\right]
$$

where, according to (30), we have $A \simeq \mathbb{Z}[S] /\left(S^{M}-1\right)$. The characters under consideration are given as follows (taking (33) as an identification):

$$
\begin{aligned}
& f_{i}=\operatorname{ch}\left(\left[V_{i}\right]\right)=\sum_{j=1}^{m_{i}} \lambda_{i, j} \stackrel{(40)}{=} \sum_{j=1}^{m_{i}} \zeta^{a_{i, j}} \pi_{1}^{a_{1, i, j}} \ldots \pi_{N}^{a_{N, i, j}} \\
& =\sum_{j=1}^{m_{i}} S^{a_{i, j}} T_{1}^{a_{1, i, j}} \ldots T_{N}^{a_{N, i, j}}, 1 \leq i \leq n .
\end{aligned}
$$

We define $\phi: A \longrightarrow \overline{\mathbb{Q}}$ by $S \mapsto 1$ and

$$
\psi: \overline{\mathbb{Q}}\left[T_{1}, T_{1}^{-1}, \ldots, T_{N}, T_{N}^{-1}\right] \hookrightarrow Q:=\overline{\mathbb{Q}}\left(T_{1}, \ldots, T_{N}\right)
$$

to be the natural inclusion. Now we show that the $\left[V_{i}\right] \in K_{0}\left(\operatorname{Rep}_{\hat{\mathbb{Z}}} K\right)$ are algebraically independent. Using Lemma 10 it suffices to show that $\psi \circ(\phi \otimes 1)(J)$ has rank $n$ over $Q$. Here $J$ is the Jacobi matrix $J=\left(\frac{\partial f_{i}}{\partial T_{j}}\right)_{1 \leq i \leq n, 1 \leq j \leq N}$. Since $n \leq N$, by (39) this is equivalent to the vectors

$$
v_{i}:=\left(\psi \circ(\phi \otimes 1)\left(\frac{\partial f_{i}}{\partial T_{j}}\right)\right)_{1 \leq j \leq N}, 1 \leq i \leq n,
$$


being linearly independent over $Q$. Let us abbreviate $x_{i, j}:=\psi \circ(\phi \otimes 1)\left(\frac{\partial f_{i}}{\partial T_{j}}\right) \in Q$, hence $v_{i}=\left(x_{i, j}\right)_{1 \leq j \leq N}$. We first prove the following claim concerning the shape of the matrix $\left(x_{i, j}\right)$.

Claim. For every $1 \leq i \leq n$ there is some $1 \leq j_{i} \leq N$ such that

$$
\begin{aligned}
x_{i^{\prime}, j_{i}} & =0 \text { for } i^{\prime}<i \text { and } \\
x_{i, j_{i}} & \neq 0 .
\end{aligned}
$$

Indeed, given $1 \leq i \leq n$ let $j_{i}$ be any integer satisfying $d_{i-1}<j_{i} \leq d_{i}$ which exists by (38). This choice guarantees that, in the notation of (41), we have

$$
\begin{aligned}
a_{j_{i}, i, l} & \geq 1 \text { for some } 1 \leq l \leq m_{i} \text { and } \\
a_{j_{i}, i^{\prime}, l} & =0 \text { for } i^{\prime}<i \text { and all } l,
\end{aligned}
$$

and the claim follows. Put a little less formally: In (41) there is, for every $i$, some variable $T_{j_{i}}$ which occurs in $f_{i}$ but does not occur in any $f_{i^{\prime}}$ with $i^{\prime}<i$. This is where (35) is used. Now the linear independence of $v_{1}, \ldots, v_{n}$ over $Q$ follows. Assume by contradiction that we are given $\alpha_{1}, \ldots, \alpha_{k} \in Q, \alpha_{k} \neq 0$, such that $\sum_{i=1}^{k} \alpha_{i} v_{i}=0$. Taking the $j_{k}$-component of this relation we get $\sum_{i=1}^{k} \alpha_{i} x_{i, j_{k}}=$ 0 in $Q$ which by (42) simplifies to $\alpha_{k} x_{k, j_{k}}=0$ with $x_{k, j_{k}} \neq 0$, hence $\alpha_{k}=0$, a contradiction. At this point the proof of the fact that the classes $\left[V_{i}\right] \in K_{0}\left(\operatorname{Rep}_{\hat{\mathbb{Z}}} K\right)$ are algebraically independent is complete.

To show that they are in fact geometrically algebraically independent, let an open subgroup of finite index $T^{\prime} \subset \hat{\mathbb{Z}}$ be given. There is an integer $L \geq 1$ such that $T^{\prime}=L \hat{\mathbb{Z}}$ and the set of eigenvalues of the topological generator $L \in T^{\prime}$ acting on $\operatorname{Res}_{T^{\prime}}^{\hat{Z}}\left(V_{i}\right)$ is $\left\{\lambda_{i, j}^{\prime}\right\}_{i, j}=\left\{\lambda_{i, j}^{L}\right\}_{i, j}$. So the homomorphism

$$
\left\langle\lambda_{i, j}\right\rangle_{1 \leq i \leq k, 1 \leq j \leq m_{i}} \longrightarrow\left\langle\lambda_{i, j}^{\prime}\right\rangle_{1 \leq i \leq k, 1 \leq j \leq m_{i}}, x \mapsto x^{L},
$$

is surjective with finite kernel and induces an isomorphism

$$
A_{k} \stackrel{\sim}{\longrightarrow}\left\langle\lambda_{i, j}^{\prime}\right\rangle_{1 \leq i \leq k, 1 \leq j \leq m_{i}} \otimes_{\mathbb{Z}} \mathbb{Q}
$$

for all $1 \leq k \leq n$. Thus the tuple $\left(T^{\prime}, \operatorname{Res}_{T^{\prime}}^{\hat{Z}}\left(V_{i}\right)\right)$ satisfies the analogue of condition (35), and by what has already been proved we see that the $\left[\operatorname{Res}_{T^{\prime}}^{\hat{\mathbb{Z}}}\left(V_{i}\right)\right] \in$ $K_{0}\left(\operatorname{Rep}_{T^{\prime}} K\right)$ are algebraically independent. As $T^{\prime}$ was arbitrary this shows that the $V_{i}$ are in fact geometrically algebraically independent and concludes the proof.

Before engaging into the slightly involved proof of Theorem 12 we remark that if in this result one replaces curves by abelian varieties one can give a quick proof as follows: The subgroup $\Gamma \subset \overline{\mathbb{Q}}^{*}$ generated by $q$-Weil numbers of weight one has infinite rank. Choosing a suitable sequence of such Weil numbers and corresponding abelian varieties (which exist by T. Honda), one obtains the result using Lemma 14 and Corollary 3 Even though $\operatorname{rk}(\Gamma)=\infty$ is well known we could not find it stated explicitly in the literature, so we record this result (which will be clear from our proof of Theorem (12) for reference.

Corollary 16. Let $q$ be a prime power. Then the subgroup of $\overline{\mathbb{Q}}^{*}$ generated by $q$-Weil numbers of weight one has infinite rank.

We now start the proof of Theorem 12. In fact, we will give a family of curves satisfying the conclusion of Theorem 12 explicitly. 
Let $\mathbb{F}_{p} \subset k$ be the prime field of $k$ and for any $a \geq 1$ let $Y_{a} / \mathbb{F}_{p}$ be the proper smooth curve which is birational to the affine plane curve given by

$$
y^{p}-y=x^{p^{a}-1} \text {. }
$$

The $Y_{a}$ are geometrically connected of genus $\frac{1}{2}\left(p^{a}-2\right)(p-1)$. The arithmetic of these curves has been studied by H. Davenport and H. Hasse [DH. Unfortunately, we cannot assert that the sequence $\left\{Y_{a}\right\}_{a \geq 1}$ satisfies the conclusion of Theorem 12 but a suitable subsequence will do. Let $T=T(p)$ be the integer determined by Lemma 19 below and let $\left\{n_{i}\right\}_{i \geq 1}$ be the sequence of prime numbers greater than $T$ ordered increasingly. Then the

$$
X_{i}:=Y_{n_{i}} \otimes_{\mathbb{F}_{p}} k, i \geq 1,
$$

are proper, smooth and geometrically connected curves over $k$, and the following result makes the existence statement of Theorem 12 explicit.

Theorem 17. The classes $\left[X_{i}\right] \in K_{0}\left(\operatorname{Var}_{k}\right)(i \geq 1)$ are algebraically independent.

For any $i \geq 1$ let us write $V_{i}:=H_{c}^{1}\left(\bar{Y}_{n_{i}}\right) \in \operatorname{Rep}_{G_{\mathbb{F}_{p}}} \mathbb{Q}_{l}$. Using Corollary [3, Theorem 17 results from the following.

Theorem 18. The classes $V_{i} \in \operatorname{Rep}_{G_{\mathbb{F}_{p}}} \mathbb{Q}_{l}(i \geq 1)$ are geometrically algebraically independent.

This will be proved using Lemma 14 for which we need to review some results concerning the Weil numbers attached to the Jacobians of the curves $Y_{a} / \mathbb{F}_{p}$.

We fix a valuation $v$ of $\overline{\mathbb{Q}}$ lying over $p$ and normalized by $v(p)=1$. We will denote by the same letter $v$ the restriction of $v$ to any subfield $K \subset \overline{\mathbb{Q}}$.

Let $F_{p}$ be the geometric Frobenius of $\mathbb{F}_{p}$. Then the eigenvalues of $F_{p}^{a}$ acting on $H_{c}^{1}\left(\bar{Y}_{a}\right)$ are given by generalized Jacobi sums

$$
\tau_{j}\left(\chi^{t}\right)^{(a)}:=-\sum_{u \in \mathbb{F}_{p}^{*} a} \chi^{t}(u) \exp \left(\frac{2 \pi i}{p} \cdot j \cdot \operatorname{tr}(u)\right), 1 \leq j \leq p-1,1 \leq t \leq p^{a}-2,
$$

where $\chi: \mathbb{F}_{p^{a}}^{*} \hookrightarrow \mathbb{C}^{*}$ is a certain faithful character of $\mathbb{F}_{p^{a}}^{*}$ (a Teichmüller lift depending on the choice of $v$; see $[\mathrm{Ma}, \S 4)$ and $\operatorname{tr}: \mathbb{F}_{p^{a}} \longrightarrow \mathbb{F}_{p}$ denotes the trace, $\mathbf{Y}$, (2).

We will abbreviate $\tau\left(\chi^{t}\right)^{(a)}:=\tau_{1}\left(\chi^{t}\right)^{(a)}$ in the following. Let $1 \leq t \leq p^{a}-2$ be given and let

$$
t=\sum_{i=0}^{a-1} j_{i} p^{i}, 0 \leq j_{i} \leq p-1,
$$

be the $p$-adic expansion of $t$ and put $\sigma(t):=\sum_{i=0}^{a-1} j_{i}$. Then [Ma], $\S 4$, gives the valuations of the generalized Jacobi sums as

$$
v\left(\tau\left(\chi^{t}\right)^{(a)}\right)=\frac{\sigma(t)}{p-1} .
$$

We will need the following lower bound on Euler's phi-function, $\phi(n):=\left|(\mathbb{Z} / n)^{*}\right|$ for $n \geq 1$.

Lemma 19. Let $p$ be a prime number. Then there is an integer $T=T(p)$ such that

$$
\frac{\phi\left(p^{n}-1\right)}{n}>\phi(p-1) \text { for all } n \geq T \text {. }
$$


Proof. This follows from the inequality, valid for any $m \geq 1$,

$$
m-1 \geq \phi(m) \geq \frac{m}{C \log \log m},
$$

with a suitable positive constant $C$. The first inequality is trivial and the second is $\underline{\mathrm{RS}}$, Theorem 15.

Proof of Theorem 18. It suffices to show that for any given $M \geq 1$ the $V_{1}, \ldots, V_{M} \in$ $\operatorname{Rep}_{G_{\mathbb{F}_{p}}} \mathbb{Q}_{l}$ are geometrically algebraically independent. So we fix some $M \geq 1$. For any $1 \leq i \leq M$ let $\left\{\lambda_{i, j}\right\}_{1 \leq j \leq\left(p^{\left.n_{i}-2\right)(p-1)}\right.} \subset \overline{\mathbb{Q}}^{*}$ be the eigenvalues of $F_{p}$ acting on $V_{i}$. Note that

$$
\left\{\lambda_{i, j}^{n_{i}}\right\}_{1 \leq j \leq\left(p^{n_{i}}-2\right)(p-1)}=\left\{\tau_{j}\left(\chi^{t}\right)^{\left(n_{i}\right)}\right\}_{1 \leq j \leq p-1,1 \leq t \leq p^{n_{i}-2}}
$$

by (43). We define $B_{0}:=\{1\} \subset \overline{\mathbb{Q}}^{*}$ and for $1 \leq k \leq M$ we define $B_{k}:=$ $\left\langle\lambda_{i, j}\right\rangle_{1 \leq i \leq k, \text { all } j} \subset \overline{\mathbb{Q}}^{*}$ to be the subgroup of $\overline{\mathbb{Q}}^{*}$ generated by the $F_{p}$-eigenvalues occurring among the $V_{1}, \ldots, V_{k}$ and $A_{k}:=B_{k} \otimes_{\mathbb{Z}} \mathbb{Q}, d_{k}:=\operatorname{dim}{ }_{\mathbb{Q}} A_{k}$. Using Lemma 14 the proof will be complete if we establish the following:

$$
\text { We have } d_{k}>d_{k-1} \text { for } k=1, \ldots, M \text {. }
$$

As $B_{k-1} \subset B_{k}$ we always have $d_{k} \geq d_{k-1}$, and we need to show that this inequality is strict. For any $n \geq 1$ we denote by $K_{n} \subset \overline{\mathbb{Q}}$ the field of $n$-th roots of unity and we fix isomorphisms $\operatorname{Gal}\left(K_{n} / \mathbb{Q}\right) \simeq(\mathbb{Z} / n)^{*}$ using a compatible system of roots of unity in $\overline{\mathbb{Q}}^{*}$. Recall that $K_{n} \cap K_{m}=K_{\operatorname{gcd}(n, m)}$ and $K_{n} K_{m}=K_{\mathrm{lcm}(n, m)}$, where gcd and $\mathrm{lcm}$ mean greatest common divisor and least common multiple, respectively. From (45) and (43) we see that

$$
\lambda_{i, j}^{n_{i}} \in K_{p^{n_{i}-1}} K_{p}=K_{p\left(p^{n_{i}}-1\right)},
$$

hence, introducing $N_{k}:=\prod_{i=1}^{k} n_{i}$ for $0 \leq k \leq M$, we have that

$$
B_{k}^{N_{k}}:=\left\{x^{N_{k}}: x \in B_{k}\right\} \subset K_{p\left(p^{\left.N_{k}-1\right)}\right.} \text { for } k=0, \ldots, M .
$$

We now prove (46) by contradiction, assuming that we are given some $1 \leq k \leq M$ such that

$$
d_{k}=d_{k-1} .
$$

As $k$ will be fixed from now on, we put $n:=n_{k}$ and $N:=N_{k-1}$ to ease the reading. The cyclotomic fields to play a role are organized in the following diagram:

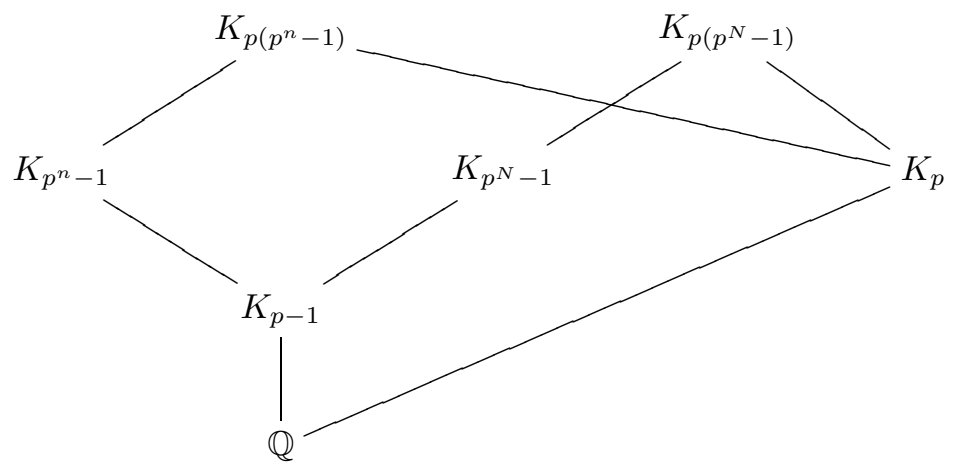

Observe that by construction $\operatorname{gcd}(n, N)=1$ ( $N$ is a product of primes different from the prime $n)$ and hence $\operatorname{gcd}\left(p^{n}-1, p^{N}-1\right)=p-1$ which implies as recalled 
above that $K_{p^{n}-1} \cap K_{p^{N-1}}=K_{p-1}$ as asserted by (50). The remaining assertions implicit in (50) are checked similarly. According to (43) there are $\lambda_{1}$ and $\lambda_{2}$ which are eigenvalues of $F_{p}$ acting on $V_{k}$ (hence $\lambda_{i} \in B_{k}$ by the definition of $B_{k}$ ) and such that

$$
\begin{aligned}
& \lambda_{1}^{n}=\tau\left(\chi^{1}\right)^{(n)}, \\
& \lambda_{2}^{n}=\tau\left(\chi^{p^{n}-2}\right)^{(n)} ;
\end{aligned}
$$

hence $\lambda_{i}^{n} \in K_{p\left(p^{n}-1\right)}$ by (43) and by [Y], (14) we have

$$
\tau_{i}:=\lambda_{i}^{n(p-1)} \in K_{p^{n}-1}, i=1,2 .
$$

The idea now is the following. We will exhibit two places $v_{1}$ and $v_{2}$ of $K_{p^{n}-1}$ lying above the same place of $K_{p-1}$ such that a suitable linear combination $x$ of $\tau_{1}$ and $\tau_{2}$ has different valuations at $v_{1}$ and $v_{2}$, and using (49) we will see that we may choose $x$ to lie in $K_{p-1}$ which will give the desired contradiction. Here are the details.

The inclusion $K_{p-1} \subset K_{p^{n}-1}$ corresponds to a surjection of Galois groups

$$
\pi:\left(\mathbb{Z} / p^{n}-1\right)^{*} \longrightarrow(\mathbb{Z} / p-1)^{*} .
$$

The decomposition group of the place $v_{1}:=v$ of $K_{p^{n}-1}$ is generated by the residue of $p$ (since $p$ is unramified in $K_{p^{n}-1}$ ) and $\pi$ factors over a surjection

$$
\bar{\pi}:\left(\mathbb{Z} / p^{n}-1\right)^{*} /\langle\bar{p}\rangle \longrightarrow(\mathbb{Z} / p-1)^{*} .
$$

The left-hand side of (53) acts simply transitively by conjugation on the set of places of $K_{p^{n}-1}$ lying above $p$, and this will be used to construct a second such place $v_{2}$. The order of the left-hand side of (53) is $\phi\left(p^{n}-1\right) / n>\phi(p-1)=\left|(\mathbb{Z} / p-1)^{*}\right|$ by Lemma 19 and our choice that $n \geq T(p)$ so we may choose

$$
1 \neq \gamma \in \operatorname{ker}(\bar{\pi}) \text {. }
$$

We denote by $v_{2}$ the place of $K_{p^{n}-1}$ conjugate by $\gamma^{-1}$ to $v_{1}$, i.e. $v_{2}(x)=$ $v_{1}(\gamma \cdot x)=v(\gamma \cdot x)$ for all $x \in K_{p^{n}-1}^{*}$. Now we compute some valuations:

$$
\begin{gathered}
v_{1}\left(\tau_{1}\right) \stackrel{(52)}{=}(p-1) v\left(\lambda_{1}^{n}\right) \stackrel{(51), \text { (44) }}{=}(p-1) \frac{\sigma(1)}{p-1}=1 \text { and } \\
v_{1}\left(\tau_{2}\right) \stackrel{(52)}{=}(p-1) v\left(\lambda_{2}^{n}\right) \stackrel{(51], \text {, (44) }}{=}(p-1) \frac{\sigma\left(p^{n}-2\right)}{p-1}=n(p-1)-1,
\end{gathered}
$$

using for the computation of $\sigma\left(p^{n}-2\right)$ that

$$
p^{n}-2=p^{n}-1-1=\sum_{i=0}^{n-1}(p-1) p^{i}-1=(p-2)+\sum_{i=1}^{n-1}(p-1) p^{i} .
$$

Let the $p$-adic expansion of $\gamma$ be given by

$$
\gamma=\sum_{i=0}^{n-1} \alpha_{i} p^{i}, 0 \leq \alpha_{i} \leq p-1
$$

Observe that

$$
\sigma(\gamma)=\sum_{i=0}^{n-1} \alpha_{i} \equiv \bar{\pi}(\gamma) \stackrel{(54)}{\equiv} 1(\bmod (p-1))
$$


If we had $\sigma(\gamma)=1$, then $\left[\mathbf{Y}\right.$, Lemma 3, ii), would imply that $\gamma \in p^{\mathbb{Z}}$, hence $\gamma=1$ in $\left(\mathbb{Z} / p^{n}-1\right)^{*} /\langle\bar{p}\rangle$, contrary to our choice (154). So we have

$$
\sigma(\gamma)=1+a(p-1) \text { for some } a \geq 1
$$

Now we compute the valuations at $v_{2}$ :

$$
\begin{aligned}
& v_{2}\left(\tau_{1}\right) \stackrel{\sqrt[52],]{=}(51]}{=}(p-1) v_{2}\left(\tau\left(\chi^{1}\right)^{(n)}\right)=(p-1) v\left(\gamma \cdot \tau\left(\chi^{1}\right)^{(n)}\right) \\
& =(p-1) v\left(\tau\left(\chi^{\gamma}\right)^{(n)}\right) \stackrel{(44)}{=}(p-1) \frac{\sigma(\gamma)}{p-1} \stackrel{(56)}{=} 1+a(p-1) .
\end{aligned}
$$

In the third term we consider $\gamma$ as an element of $\operatorname{Gal}\left(K_{p^{n}-1} / \mathbb{Q}\right)$, and the second equality follows from our definition of $v_{2}$. The third equality uses $\gamma \cdot \tau\left(\chi^{t}\right)^{(n)}=$ $\tau\left(\chi^{\gamma t}\right)^{(n)}$ which follows from (43). Similarly we have

$$
\begin{array}{r}
v_{2}\left(\tau_{2}\right) \stackrel{\sqrt[52)]{=}, \sqrt{51}}{=}(p-1) v_{2}\left(\tau\left(\chi^{p^{n}-2}\right)^{(n)}\right)=(p-1) v\left(\tau\left(\chi^{\gamma\left(p^{n}-2\right)}\right)^{(n)}\right) \\
=(p-1) v\left(\tau\left(\chi^{-\gamma}\right)^{(n)}\right) \stackrel{(44)}{=}(p-1) \frac{\sigma(-\gamma)}{p-1}=\sigma(-\gamma) \stackrel{(*)}{=} n(p-1)-\sigma(\gamma) \\
\stackrel{(56)}{=} n(p-1)-(1+a(p-1))=(n-a)(p-1)-1 .
\end{array}
$$

In this sequence of equalities, we would like to justify $(*)$, which is an elementary property of the function $\sigma$ : From (64) we get the $p$-adic expansion of $-\gamma$ :

$$
-\gamma=-\sum_{i=0}^{n-1} \alpha_{i} p^{i} \stackrel{\left(\bmod \underline{p}^{n}-1\right)}{=} p^{n}-1-\sum_{i=0}^{n-1} \alpha_{i} p^{i}=\sum_{i=0}^{n-1}\left(p-1-\alpha_{i}\right) p^{i}
$$

hence

$$
\sigma(\gamma)+\sigma(-\gamma)=\sum_{i=0}^{n-1} \alpha_{i}+\sum_{i=0}^{n-1}\left(p-1-\alpha_{i}\right)=n(p-1)
$$

as used in $\left(^{*}\right)$. To sum up, we have computed the matrix

$$
X:=\left(\begin{array}{ll}
v_{1}\left(\tau_{1}\right) & v_{1}\left(\tau_{2}\right) \\
v_{2}\left(\tau_{1}\right) & v_{2}\left(\tau_{2}\right)
\end{array}\right)=\left(\begin{array}{cc}
1 & n(p-1)-1 \\
1+a(p-1) & (n-a)(p-1)-1
\end{array}\right)
$$

and compute its determinant

$$
\begin{array}{r}
\operatorname{det}(X)=(n-a)(p-1)-1-(1+a(p-1))(n(p-1)-1) \\
=(n-a)(p-1)-1-\left(n(p-1)-1+a n(p-1)^{2}-a(p-1)\right) \\
=-a n(p-1)^{2} \neq 0,
\end{array}
$$

because $a \neq 0$ by (56). If $\mu, \nu \in \mathbb{Z}$ are given and we put $x:=\tau_{1}^{\mu} \tau_{2}^{\nu} \in K_{p^{n}-1}$ we have

$$
\left(\begin{array}{l}
v_{1}(x) \\
v_{2}(x)
\end{array}\right)=X\left(\begin{array}{c}
\mu \\
\nu
\end{array}\right)
$$

By (57) there is some integer $R \geq 1$ and $\mu, \nu \in \mathbb{Z}$ such that $X\left(\begin{array}{c}\mu \\ \nu\end{array}\right)=\left(\begin{array}{l}R \\ 0\end{array}\right)$, so if we put $x:=\tau_{1}^{\mu} \tau_{2}^{\nu}$ for these particular values of $\mu$ and $\nu$ we get $x \in K_{p^{n}-1}$ with

$$
v_{1}(x)=R \geq 1 \text { and } v_{2}(x)=0 .
$$

Note that for the restrictions of $v_{1}$ and $v_{2}$ to $K_{p-1}$ we have

$$
\left.v_{1}\right|_{K_{p-1}}=\left.v_{2}\right|_{K_{p-1}}
$$


because the restriction of $\gamma \in \operatorname{Gal}\left(K_{p^{n}-1} / \mathbb{Q}\right)$ to $K_{p-1}$ is trivial, i.e. $\bar{\pi}(\gamma)=1$; cf. (54). Now we can finally derive a contradiction. By (49) we know that $B_{k} / B_{k-1}$ is torsion. As $\lambda_{1}, \lambda_{2} \in B_{k}$ we find an integer $S \geq 1$ with

$$
\lambda_{i}^{S} \in B_{k-1}^{N} \stackrel{\sqrt[48]{\complement}}{\complement} K_{p\left(p^{N}-1\right)}, i=1,2
$$

(recall that $N=N_{k-1}$ ), and furthermore $x^{S}=\left(\tau_{1}^{\mu} \tau_{2}^{\nu}\right)^{S} \stackrel{\sqrt[52]{=}}{=}\left(\lambda_{1}^{\mu} \lambda_{2}^{\nu}\right)^{n(p-1) S}$, so $x^{S} \in K_{p^{n}-1}$ by (52) and $x^{S} \in K_{p\left(p^{N}-1\right)}$ by (60), from which we conclude that

$$
x^{S} \in K_{p^{n}-1} \cap K_{p\left(p^{N}-1\right)} \stackrel{\sqrt{500}}{=} K_{p-1} .
$$

This implies $S R \stackrel{[58]}{=} v_{1}\left(x^{S}\right) \stackrel{[61],[59]}{=} v_{2}\left(x^{S}\right) \stackrel{[58]}{=} 0$, a contradiction which concludes the proof.

Proof of Theorem 13. We can assume that the given set of elliptic curves $\left\{E_{i}\right\}_{i \in I}$ $=\left\{E_{1}, \ldots, E_{n}\right\}$ is finite. We will show that the classes of $V_{i}:=H_{c}^{1}\left(\overline{E_{i}}\right)$ in $K_{0}\left(\operatorname{Rep}_{G_{k}} \mathbb{Q}_{l}\right)$ are algebraically independent which is sufficient by Corollary 3 . We consider the subring $R:=\mathbb{Z}\left[\left[V_{i}\right]\right] \subset K_{0}\left(\operatorname{Rep}_{G_{k}} \mathbb{Q}_{l}\right)$ generated by the $\left[V_{i}\right]$. For any finite place $v$ of $k$ with residue field $k(v)$ at which all the $E_{i}$ have good reduction, we have a canonical homomorphism $\phi_{v}: R \longrightarrow K_{0}\left(\operatorname{Rep}_{G_{k}(v)} \mathbb{Q}_{l}\right)$ because all $V_{i}$ are unramified at $v$. We are going to construct such a place $v$ so that the $\phi_{v}\left(\left[V_{i}\right]\right)$ are algebraically independent. For $A:=E_{1} \times \cdots \times E_{n}$ we have $H_{c}^{1}(\bar{A}) \simeq \bigoplus_{i=1}^{n} V_{i}$ and denote by $G_{l} \subset \operatorname{Gl}\left(H_{c}^{1}(\bar{A})\right)\left(\mathbb{Q}_{l}\right)$ the closure of the image of Galois. Since End $_{\bar{k}}\left(E_{i}\right)=\mathbb{Z}$ we know that the Hodge group $\operatorname{Hdg}\left(H^{1}\left(E_{i}\right)\right)$ is isogeneous to $\mathrm{Sl}_{2}$. As the $E_{i}$ are pairwise non-isogeneous we have that $\operatorname{Hgd}\left(H^{1}(A)\right)$ is isogeneous to $\mathrm{Sl}_{2}^{n}$, and hence the Mumford-Tate group $\operatorname{MT}\left(H^{1}(A)\right)$ has rank $n+1$. Now observe that the Mumford-Tate conjecture is known to be true for $A / k$ : For $n=1$ it is a celebrated result of J-P. Serre, and one reduces to this case using $[\mathrm{R}$, Lemma on page 790. We conclude that $G_{l}$ has rank $n+1$. By [S5] we find a finite place $v$ of $k$ of good reduction for all $E_{i}$ and such that the corresponding Frobenius torus $T_{v} \subset G_{l}^{0}$ is maximal, i.e. $T_{v}$ has rank $n+1$. But this rank is nothing other than $\operatorname{dim}_{\mathbb{Q}} A_{n}$ in the notation of Lemma 15 (applied to the reductions at $v$ of the $E_{i}$ ), and using this Lemma we conclude that the $\phi_{v}\left(\left[V_{i}\right]\right)$ are indeed algebraically independent as was to be shown.

3.2. Using a result of Skolem. In the previous subsection we used a Tannakian argument to establish the algebraic independence of $l$-adic Galois representations given by the cohomology of suitable varieties. In this subsection we approach the same goal using a lemma of Skolem on the shape of the power series expansion of rational functions together with the rationality properties given in Section 2.3. The possibility of this was suggested by reading a note of J-P. Serre [S2].

Let $k$ be a finite field. We will use the motivic measure from Proposition 7 .

$$
\mu_{k}: K_{0}\left(\operatorname{Var}_{k}\right) \longrightarrow \mathcal{R}(\mathbb{Q})[T],
$$

to give sufficient conditions for the classes of given varieties over $k$ to be algebraically independent in $K_{0}\left(\operatorname{Var}_{k}\right)$.

The main observation is that, if there is a non-trivial algebraic relation among the motivic measures of some varieties, then, at least after a finite extension of the base field, there will be an irreducible relation; see Section 3.3 . 
For $\nu \geq 1$ we denote by $k_{\nu}$ the unique extension of degree $\nu$ of $k$ inside some fixed algebraic closure of $k$.

\subsection{Irreducible equations.}

Theorem 20. Let $k$ be a finite field and let $X_{1}, \ldots, X_{n} / k(n \geq 1)$ be separated and of finite type and assume that $\mu_{k}\left(\left[X_{1}\right]\right), \ldots, \mu_{k}\left(\left[X_{n}\right]\right)$ are algebraically dependent in $\mathcal{R}(\mathbb{Q})[T]$. Then there are $M \geq 1$ and an irreducible polynomial $G \in \mathbb{Z}\left[T_{1}, \ldots, T_{n}\right]$ such that

$$
G\left(\mu_{k_{M}}\left(\left[X_{1} \times_{k} k_{M}\right]\right), \ldots, \mu_{k_{M}}\left(\left[X_{n} \times_{k} k_{M}\right]\right)\right)=0 .
$$

Remark. This result is non-trivial even for $n=1$ because $\mathcal{R}(\mathbb{Q})[T]$ contains zero divisors. In the remark following the proof of Theorem 25 we give an example showing the necessity of allowing the finite extension $k \subset k_{M}$ in Theorem 20.

The starting point of the proof of Theorem 20 is a result due to Skolem which we now explain. For brevity, we will call a subset $X \subset \mathbb{N} \operatorname{good}$ if it is the union of a finite set and finitely many arithmetic progressions for a single modulus, i.e. $X$ is good if there are $\Sigma \subset \mathbb{N}$ finite, $M \geq 1$ and $I \subset\{0, \ldots, M-1\}$ such that

$$
X=\Sigma \bigcup_{i \in I}(i+M \mathbb{N}) \text {. }
$$

The collection of good subsets is stable under finite union and finite intersection. Skolem's result is the following $\mathrm{S} 2$.

Proposition 21. Let $f \in \mathbb{Q}(t)$ be a rational function with power series expansion $f=\sum_{\nu \in \mathbb{Z}} a_{\nu} t^{\nu}$. Then the set $\left\{\nu \geq 0: a_{\nu}=0\right\}$ is good.

We will use the following consequence of Skolem's result.

Corollary 22. For $f \in \mathcal{R}(\mathbb{Q})$ the set $\left\{\nu \geq 1: \partial_{\nu}(f)=0\right\}$ is good.

Indeed, for $f \in \mathcal{R}(\mathbb{Q})$ we have that $\operatorname{dlog}(f)$ is a rational function to which we apply Proposition 21. As the collection of good subsets is stable under finite intersection, we also have the following.

Corollary 23. For $F \in \mathcal{R}(\mathbb{Q})[T]$ the set $\left\{\nu \geq 1: \partial_{\nu}(F)=0\right.$ in $\left.\mathbb{Q}[T]\right\}$ is good.

Corollary 23 follows from Corollary 22 applied to the coefficients of $F$. By abuse of notation, in Corollary 23, we have written $\partial_{\nu}: \mathcal{R}(\mathbb{Q})[T] \rightarrow \mathbb{Q}[T]$ for the map derived from $\partial_{\nu}: \mathcal{R}(\mathbb{Q}) \subset \Lambda(\mathbb{Q}) \rightarrow \mathbb{Q}$ by sending $T \mapsto T$.

Proof of Theorem 20 . By assumption we have some $0 \neq H \in \mathbb{Z}\left[T_{1}, \ldots, T_{n}\right]$ such that

$$
H\left(\mu_{k}\left(\left[X_{1}\right]\right), \ldots, \mu_{k}\left(\left[X_{n}\right]\right)\right)=0 \text { in } \mathcal{R}(\mathbb{Q})[T] .
$$

Decompose $H=\prod_{i=1}^{N} H_{i}$ into a product of irreducible $H_{i} \in \mathbb{Z}\left[T_{1}, \ldots, T_{n}\right]$ and consider

$$
X_{i}:=\left\{\nu \geq 1 \mid \partial_{\nu}\left(H_{i}\left(\mu_{k}\left(\left[X_{1}\right]\right), \ldots, \mu_{k}\left(\left[X_{n}\right]\right)\right)\right)=0\right\}, 1 \leq i \leq N .
$$

By Corollary 23 the $X_{i} \subset \mathbb{N}$ are good and for fixed $\nu \geq 1$ we have

$0 \stackrel{\sqrt[62]{=}}{=} \partial_{\nu}\left(H\left(\mu_{k}\left(\left[X_{1}\right]\right), \ldots, \mu_{k}\left(\left[X_{n}\right]\right)\right)\right)=\prod_{i=1}^{N} \partial_{\nu}\left(H_{i}\left(\mu_{k}\left(\left[X_{1}\right]\right), \ldots, \mu_{k}\left(\left[X_{n}\right]\right)\right)\right)$ in $\mathbb{Q}[T]$.

As $\mathbb{Q}[T]$ is an integral domain this implies that $\bigcup_{i=1}^{N} X_{i}=\mathbb{N}$. 
We claim that this implies the existence of $1 \leq i_{0} \leq N$ and $M \geq 1$ such that $M \mathbb{N} \subset X_{i_{0}}$. Indeed, assume that this is not the case and write

$$
X_{i}=\Sigma_{i} \bigcup_{j \in I_{i}}\left(j+M_{i} \mathbb{N}\right), i=1, \ldots, N,
$$

for suitable finite sets $\Sigma_{i}$, integers $M_{i} \geq 1$ and subsets $I_{i} \subset\left\{0, \ldots, M_{i}-1\right\}$. Then $0 \notin I_{i}$ for $i=1, \ldots, N$. Consider a positive multiple $x$ of the product $M_{1} \ldots M_{N}$ so large that $x \notin \bigcup \Sigma_{i}$. As $\mathbb{N}=\bigcup X_{i}$ we find $i_{0}$ and $j \in I_{i_{0}}$ such that $x \in j+M_{i_{0}} \mathbb{N}$ hence $x \equiv j\left(\bmod M_{i_{0}}\right)$ and $x \equiv 0\left(\bmod M_{i_{0}}\right)$, a contradiction because $j \in I_{i_{0}}$, hence $j \not \equiv 0\left(\bmod M_{i_{0}}\right)$, so there do indeed exist $M \geq 1$ and $i_{0}$ such that $M \mathbb{N} \subset X_{i_{0}}$. We will see that $G:=H_{i_{0}}$ and $M$ satisfy the requirements of Theorem 20. By construction, $G$ is irreducible, and it remains to be checked that

$$
G\left(\mu_{k_{M}}\left(\left[X_{1} \times_{k} k_{M}\right]\right), \ldots, \mu_{k_{M}}\left(\left[X_{n} \times_{k} k_{M}\right]\right)\right)=0 .
$$

Equivalently, as $\bigcap_{\nu \geq 1} \operatorname{ker}\left(\partial_{\nu}: \mathcal{R}(\mathbb{Q})[T] \rightarrow \mathbb{Q}[T]\right)=0$ we need to show that for all $\nu \geq 1$

$$
\partial_{\nu}\left(G\left(\mu_{k_{M}}\left(\left[X_{1} \times_{k} k_{M}\right]\right), \ldots, \mu_{k_{M}}\left(\left[X_{n} \times_{k} k_{M}\right]\right)\right)\right)=0 .
$$

Using (19) and $\partial_{\nu} F_{M}=\partial_{\nu M}$ [DG], V, $\S 5,2.6$, we obtain

$$
\begin{aligned}
& =G\left(\partial_{\nu}\left(\mu_{k_{M}}\left(\left[X_{1} \times_{k} k_{M}\right]\right)\right), \ldots, \partial_{\nu}\left(\mu_{k_{M}}\left(\left[X_{n} \times_{k} k_{M}\right]\right)\right)\right) \\
& =G\left(\partial_{\nu}\left(F_{M}\left(\mu_{k}\left(\left[X_{1}\right]\right)\right)\right), \ldots, \partial_{\nu}\left(F_{M}\left(\mu_{k}\left(\left[X_{n}\right]\right)\right)\right)\right) \\
& =\partial_{\nu M}\left(G\left(\mu_{k}\left(\left[X_{1}\right]\right), \ldots, \mu_{k}\left(\left[X_{n}\right]\right)\right)\right) .
\end{aligned}
$$

However, $\nu M \in M \mathbb{N} \subset X_{i_{0}}$, so this vanishes by construction as $G=H_{i_{0}}$; see (63).

3.4. Zero-dimensional varieties. Let $k$ be a finite field. Before applying the results of Section 3.3 we include in this subsection a digression on the subring of $K_{0}\left(\operatorname{Var}_{k}\right)$ generated by zero-dimensional varieties.

Generally speaking, the difficulty of the problem of deciding whether the classes of given varieties $\left[X_{1}\right], \ldots,\left[X_{n}\right]$ are algebraically independent in $K_{0}\left(\operatorname{Var}_{k}\right)$ grows quite rapidly with $n$. For $n=1$ a complete answer can be given.

Theorem 24. Let $k$ be a finite field and let $X / k$ be separated and of finite type. Then the following are equivalent:

1) $[X] \in K_{0}\left(\operatorname{Var}_{k}\right)$ is algebraically dependent.

2) $\quad[X]$ is integral over $\mathbb{Z}$.

$$
\text { 3) } \quad \operatorname{dim}(X)=0 \text {. }
$$

Condition 1) means that $[X]$ satisfies some polynomial with coefficients in $\mathbb{Z}$ but this polynomial does not have to be monic. So, a priori, condition 2) is stronger than condition 1). Let $\tilde{\mathbb{Z}} \subset K_{0}\left(\operatorname{Var}_{k}\right)$ denote the integral closure of $\mathbb{Z}$ inside $K_{0}\left(\operatorname{Var}_{k}\right)$ and let $S \subset K_{0}\left(\operatorname{Var}_{k}\right)$ be the subring generated by the classes of zero-dimensional varieties. By Theorem 24 we have $S \subset \tilde{\mathbb{Z}}$ and even more, for all $X / k$, if $[X] \in \tilde{\mathbb{Z}}$, then $[X] \in S$. Note that this does not imply $\tilde{\mathbb{Z}} \subset S$ because an element of $K_{0}\left(\operatorname{Var}_{k}\right)$ is in general only a formal difference of varieties.

In fact, I do not know whether $S \subset \tilde{\mathbb{Z}}$ is an equality.

One can give a simple presentation of the ring $S$; see Theorem 25 below. 
Proof of Theorem 24. We start by showing that 3) implies 2). Let $X / k$ be zero dimensional, i.e. $X=\operatorname{Spec}(A)$ for some Artinian $k$-algebra $A$. As $[X]=\left[X^{\text {red }}\right]$ we can assume that $A$ is reduced, i.e. $A=\bigoplus L_{i}$ for $L_{i} \mid k$ finite field extensions. Then $[X]=\sum\left[\operatorname{Spec}\left(L_{i}\right)\right]$, and we are reduced to showing that for a finite extension field $k \subset L$ we have $[\operatorname{Spec}(L)] \in \tilde{\mathbb{Z}}$. But, if $d=[L: k]$, then

$$
\begin{gathered}
{[\operatorname{Spec}(L)]^{2}-d[\operatorname{Spec}(L)]=\left[\operatorname{Spec}\left(L \otimes_{k} L\right)\right]-d[\operatorname{Spec}(L)]} \\
=\left[\operatorname{Spec}\left(L^{\oplus d}\right)\right]-d[\operatorname{Spec}(L)]=0 .
\end{gathered}
$$

We used that $L \mid k$ is Galois to have $L \otimes_{k} L \simeq L^{\oplus d}$ as $k$-algebras.

That 2) implies 1) is trivial, and we now show that 1 ) implies 3 ). Let $X / k$ be separated and of finite type and such that $[X]$ is algebraically dependent. We need to show that $\operatorname{dim}(X)=0$. As $[X]=\left[X^{\text {red }}\right]$ and $\operatorname{dim}(X)=\operatorname{dim}\left(X^{\text {red }}\right)$ we assume that $X$ is reduced. As $k$ is perfect this implies that the smooth locus of $X / k$ is (open and) dense. This will be used later, but first we exploit the assumption that $[X]$ is algebraically dependent. There is $0 \neq F \in \mathbb{Z}\left[T_{1}\right]$ with $F([X])=0$. Let us consider $f:=\mu_{k}([X]) \in \mathcal{R}(\mathbb{Q})[T]$. For any $\nu \geq 1$ we have

$$
F\left(\partial_{\nu}(f)\right)=F\left(\partial_{\nu}\left(\mu_{k}([X])\right)\right)=\left(\partial_{\nu} \circ \mu_{k}\right)(F([X]))=0 .
$$

As $\partial_{\nu}(f)$ lies in $\mathbb{Q}[T]$ and $F \neq 0$, this implies that $\partial_{\nu}(f)$ is a constant, i.e. $\frac{d}{d T}\left(\partial_{\nu}(f)\right)$ $=0$. Then also

$$
\partial_{\nu}\left(\frac{d f}{d T}\right)=\frac{d}{d T}\left(\partial_{\nu}(f)\right)=0 \text { for all } \nu \geq 1
$$

and using $\bigcap_{\nu \geq 1}\left(\operatorname{ker}\left(\partial_{\nu}: \mathcal{R}(\mathbb{Q})[T] \rightarrow \mathbb{Q}[T]\right)\right)=0$ we get $\frac{d f}{d T}=0$, i.e. $f$ itself is a constant, $f \in \mathcal{R}(\mathbb{Q})$. Now assume by contradiction that $d:=\operatorname{dim}(X) \geq 1$. The coefficient of $T^{2 d}$ in $f$ is, by definition of $\mu_{k}$, the image under $\chi_{q}: K_{0}\left(\operatorname{Rep}_{G_{k}} \mathbb{Q}_{l}\right) \hookrightarrow$ $\Lambda\left(\mathbb{Q}_{l}\right)$ of the virtual Galois representation $\sum_{j}(-1)^{j}\left[\operatorname{Gr}_{2 d}^{W} H_{c}^{j}(\bar{X})\right]$, and this virtual representation is zero because $f$ is a constant and $2 d>0$, i.e.

$$
\sum_{j}(-1)^{j}\left[\operatorname{Gr}_{2 d}^{W} H_{c}^{j}(\bar{X})\right]=0 .
$$

From [D3, 3.3.4, we have

$$
\begin{array}{r}
\operatorname{Gr}_{2 d}^{W} H_{c}^{j}(\bar{X})=0 \text { for } j \neq 2 d \text { and } \\
\operatorname{Gr}_{\nu}^{W} H_{c}^{2 d}(\bar{X})=0 \text { for } \nu \neq 2 d .
\end{array}
$$

From (65) and (66) we get $\operatorname{Gr}_{2 d}^{W} H_{c}^{2 d}(\bar{X})=0$ which by (67) implies

$$
H_{c}^{2 d}(\bar{X})=0 .
$$

By the introductory remarks concerning the smooth locus of $X / k$ we can choose $U \subset X$ open, smooth and purely $d$-dimensional such that the dimension of $X-U$ is strictly less than $d$ ( $U$ need not be dense in $X$; it may be chosen as the union of the smooth loci of the irreducible components of $X$ of dimension $d$ ). The last condition assures that the natural map $H_{c}^{2 d}\left(\bar{U}, \mathbb{Q}_{l}\right) \rightarrow H_{c}^{2 d}\left(\bar{X}, \mathbb{Q}_{l}\right)$ is an isomorphism, hence (68) gives $H_{c}^{2 d}\left(\bar{U}, \mathbb{Q}_{l}\right)=0$. Poincaré duality gives $H^{0}\left(\bar{U}, \mathbb{Q}_{l}\right)=0$ which is a contradiction because $\operatorname{dim}_{\mathbb{Q}_{l}} H^{0}\left(\bar{U}, \mathbb{Q}_{l}\right)$ is the number of connected components of $\bar{U}$ and $U \neq \emptyset$.

We now give a presentation of the subring $S \subset K_{0}\left(\operatorname{Var}_{k}\right)$ generated by zerodimensional varieties. From the proof of Theorem [24, 3) $\Rightarrow 2$ ), we know that $S$ is 
generated by the classes of finite field extensions of $k$. Putting $x_{\nu}:=\left[\operatorname{Spec}\left(k_{\nu}\right)\right] \in$ $K_{0}\left(\operatorname{Var}_{k}\right)(\nu \geq 1)$ we have

$$
S=\mathbb{Z}\left[x_{\nu}: \nu \geq 1\right] \subset K_{0}\left(\operatorname{Var}_{k}\right) .
$$

For $\mu, \nu \geq 1$ we write $d(\mu, \nu)$ (resp. $m(\mu, \nu)$ ) for the greatest common divisor (resp. the least common multiple) of $\mu$ and $\nu$. Computing tensor products of finite extensions of finite fields, one checks that $x_{\mu} x_{\nu}=d(\mu, \nu) x_{m(\mu, \nu)}$, and we get a surjective ring homomorphism

$$
\phi: \mathbb{Z}\left[T_{\nu}: \nu \geq 1\right] /\left(T_{\nu} T_{\mu}-d(\mu, \nu) T_{m(\mu, \nu)}: \mu, \nu \geq 1\right) \longrightarrow S, T_{\nu} \mapsto x_{\nu} .
$$

Theorem 25. The above $\phi$ is an isomorphism. In particular, the $x_{\nu}(\nu \geq 2)$ are pairwise distinct zero divisors in $K_{0}\left(\operatorname{Var}_{k}\right)$.

Remark. The existence of zero divisors in $K_{0}\left(\operatorname{Var}_{k}\right)$ was first addressed by B. Poonen in $[\mathrm{P}$ where he showed their existence for $k$ of characteristic zero. One can show that for any finitely generated field $k$ the images of the classes of the nontrivial finite Galois extensions of $k$ in $\left.\left.K_{0}\left(\operatorname{Var}_{k}\right) \llbracket \mathbb{A}_{k}^{1}\right]^{-1}\right]$ are an infinite set of zero divisors.

Proof of Theorem 25. The left-hand side of (69) is a free $\mathbb{Z}$-module on the set $\left\{T_{\nu}\right.$ : $\nu \geq 1\}$. To show that $\phi$ is injective we use that, for all $n \geq 1$, sending $[X] \mapsto\left|X\left(k_{n}\right)\right|$ defines a motivic measure $\psi_{n}: K_{0}\left(\operatorname{Var}_{k}\right) \rightarrow \mathbb{Z}$ and that, writing $X_{\nu}:=\operatorname{Spec}\left(k_{\nu}\right)$, we have

$$
\begin{array}{r}
\left|X_{\nu}\left(k_{n}\right)\right|=\nu, \text { if } \nu \text { divides } n, \\
\left|X_{\nu}\left(k_{n}\right)\right|=0, \text { otherwise, }
\end{array}
$$

for all $\nu, n \geq 1$. Now assume that $\phi\left(\sum_{\nu \geq 1} a_{\nu} T_{\nu}\right)=\sum_{\nu \geq 1} a_{\nu}\left[X_{\nu}\right]=0$ for some $a_{\nu} \in \mathbb{Z}$, almost all zero. We need to show that $a_{\nu}=0$ for all $\nu$. For any $n \geq 1$ we have

$\left(*_{n}\right) \quad 0=\psi_{n}\left(\sum_{\nu \geq 1} a_{\nu}\left[X_{\nu}\right]\right)=\sum_{\nu \geq 1} a_{\nu}\left|X_{\nu}\left(k_{n}\right)\right|=\sum_{\nu \mid n} a_{\nu} \nu$.

To show that all $a_{\nu}$ are zero we proceed by induction on the number of prime divisors, counting multiplicity, of $\nu$.

For $\nu=1$ we have $(*)_{1}: a_{1}=0$.

For $\nu \neq 1$ we have from $(*)_{\nu}$ that $\sum_{d \mid \nu} a_{d} d=0$. By induction hypothesis we have $a_{d}=0$ for all proper divisors $d$ of $\nu$, hence $a_{\nu} \nu=0$ and $a_{\nu}=0$.

This shows that $\phi$ is injective, hence an isomorphism. To prove the last claim of Theorem 25 we use that, since $\phi$ is an isomorphism, $S$ is $\mathbb{Z}$-free on the set $\left\{x_{\nu}: \nu \geq 1\right\}$ and in particular that the $x_{\nu}$ are all distinct. Finally, for $\nu \geq 2$, $0=x_{\nu}^{2}-\nu x_{\nu}=x_{\nu}\left(x_{\nu}-\nu\right)$. As $x_{\nu}-\nu=x_{\nu}-\nu x_{1} \neq 0$ (note that $x_{1}=1$ ) we see that $x_{\nu}$ is a zero divisor.

Remark. The above considerations suggest a simple example illustrating Theorem 20. We take $n:=1, X_{1}:=\operatorname{Spec}\left(k_{2}\right)$ in this result and compute $\mu_{k}\left(\left[X_{1}\right]\right) \in \mathcal{R}(\mathbb{Q})[T]$. We have $H_{c}^{i}\left(\bar{X}_{1}\right)=0$ for $i \neq 0$ and $H_{c}^{0}\left(\bar{X}_{1}\right)$ is two dimensional with geometric Frobenius $F_{k}$ acting by exchanging the vectors of a (fixed) basis. This gives for $\nu \geq 1$

$$
c_{\nu}:=\operatorname{tr}\left(F_{k}^{\nu} \mid H_{c}^{0}\left(\bar{X}_{1}\right)\right)=2 \text { for } \nu \text { even }
$$


and $c_{\nu}=0$ for $\nu$ odd. For arbitrary $G \in \mathbb{Z}\left[T_{1}\right]$ we have $G\left(\mu_{k}\left(\left[X_{1}\right]\right)\right)=0$ if and only if $G\left(\partial_{\nu}\left(\mu_{k}\left(\left[X_{1}\right]\right)\right)\right)=0$ for all $\nu \geq 1$, and from the above computation of the cohomology of $X_{1}$ and Proposition 8 i $)$ we get that $\partial_{\nu}\left(\mu_{k}\left(\left[X_{1}\right]\right)\right) \in \mathbb{Q}[T]$ is in fact a constant equal to $c_{\nu}$. So $G\left(\mu_{k}\left(\left[X_{1}\right]\right)\right)=0$ is equivalent to $G\left(c_{\nu}\right)=0$ for all $\nu \geq 1$. From (70) we see that the assertion of Theorem 20 holds with $M=2$ and $G=T_{1}-2$.

However, there is no irreducible polynomial $G \in \mathbb{Z}\left[T_{1}\right]$ satisfying $G\left(\mu_{k}\left(\left[X_{1}\right]\right)\right)=0$ because such a $G$ must, by (70), satisfy $G(0)=G(2)=0$ and hence must be divisible by $T(T-2)$.

This shows that in Theorem 20 one has to allow for a finite extension of the base field in order to find an irreducible relation.

3.5. Two curves. Let $k$ be a finite field. In this subsection by a curve over $k$ we shall always mean a proper, smooth and geometrically connected curve. We are going to treat in detail the following special case of the problem of algebraic independence in $K_{0}\left(\operatorname{Var}_{k}\right)$. Let $X$ and $Y$ be curves over $k$. When are $[X]$ and $[Y]$ algebraically independent in $K_{0}\left(\operatorname{Var}_{k}\right)$, i.e. when is the subring $\mathbb{Z}[[X],[Y]] \subset$ $K_{0}\left(\operatorname{Var}_{k}\right)$ a polynomial ring in the variables $[X]$ and $[Y]$ ? We will see that this is the case for a generic pair $(X, Y)$.

First we define special curves which will turn out to be those for which our methods can shed no light on the above question. A curve $X / k$ is special if i) the degree of $k$ over its prime field is even: $k=\mathbb{F}_{q}, q=p^{2 n}(n \geq 1)$ and ii) all eigenvalues of the geometric Frobenius attached to $k$ acting on $H_{c}^{1}(\bar{X})$ are equal to $+q^{1 / 2}$. Note that by i) $+q^{1 / 2}$ is a rational integer so ii) is meaningful. Being special is not a property of the curve alone but depends on the base field, too. The projective line is special over any $k$ satisfying condition i). An elliptic curve $X / k$ becomes special after a finite extension of the base field if and only if it is supersingular. If $X / k$ is special, then the Jacobian of $X$ is $k$-isogeneous to the power of a super-singular elliptic curve, and we see that most curves of genus at least one are non-special. The main result of this subsection is the following.

Theorem 26. Let $X_{1}$ and $X_{2}$ be proper, smooth and geometrically connected curves over the finite field $k$ such that $\left[X_{1}\right]$ and $\left[X_{2}\right]$ are algebraically dependent in $K_{0}\left(\operatorname{Var}_{k}\right)$. Then there is a finite extension $k \subset K$ such that at least one of the following holds:

1) Both $X_{1} \times_{k} K / K$ and $X_{2} \times_{k} K / K$ are special.

2) The Jacobians of $X_{1}$ and $X_{2}$ are $K$-isogeneous.

Corollary 27. If $E / k$ is an ordinary elliptic curve, then $\left[\mathbb{P}^{1}\right]$ and $[E]$ are algebraically independent in $K_{0}\left(\operatorname{Var}_{k}\right)$.

This is clear using Theorem 26 and the discussion preceding it. Theorem 26 can be applied to the following problem: It is difficult to exhibit relations in $K_{0}\left(\operatorname{Var}_{k}\right)$. One such relation, which follows directly from the definition, is that if $X \longrightarrow Y$ is a Zariski-locally trivial fibre bundle with fibre $G$, then $[X]=[G][Y]$ in $K_{0}\left(\operatorname{Var}_{k}\right)$. One might hope for a similar relation for more general $X \longrightarrow Y$, e.g. étale covers. However:

Corollary 28. Let $X / k$ be a curve of genus at least two which does not become special after any finite extension of $k$. If $Y \longrightarrow X$ is a non-trivial finite étale cover, then $[X]$ and $[Y]$ are algebraically independent in $K_{0}\left(\operatorname{Var}_{k}\right)$. 
Proof. By the assumption made on $X$, condition 1) of Theorem 26 is excluded. The Hurwitz formula implies that the genus of $Y$ is strictly larger than the genus of $X$, excluding condition 2) of Theorem 26. Hence Theorem 26] implies the algebraic independence of $[X]$ and $[Y]$.

Remark. Rephrasing Theorem 26 we have: If for two given curves $X_{1}, X_{2} / k$ none of 1) or 2) from Theorem 26 holds after any finite extension of $k$, then $\left[X_{1}\right]$ and $\left[X_{2}\right]$ are algebraically independent. We will in fact show that in this situation $\mu_{k}\left(\left[X_{1}\right]\right)$ and $\mu_{k}\left(\left[X_{2}\right]\right)$ are algebraically independent in $\mathcal{R}(\mathbb{Q})[T]$. We have seen in Corollary 3 that for the algebraic independence of the $\mu_{k}\left(\left[X_{i}\right]\right)$ it is sufficient that $\left[H_{c}^{1}\left(\bar{X}_{1}\right)\right]$ and $\left[H_{c}^{1}\left(\bar{X}_{2}\right)\right]$ are algebraically independent in $K_{0}\left(\operatorname{Rep}_{G_{k}} \mathbb{Q}_{l}\right)$. The following example shows that Theorem 26] is sharper than just the algebraic independence of $H^{1}$ 's. Let $X_{2} / k$ be a curve such that its Jacobian is $k$-isogeneous to the square of an ordinary elliptic curve $X_{1} / k$ (such a curve exists for suitable $k$ by [BDS], Theorem $2)$. Then $H_{c}^{1}\left(\bar{X}_{2}\right) \simeq H_{c}^{1}\left(\bar{X}_{1}\right)^{\oplus 2}$ as $G_{k}$-modules and so $\left[H_{c}^{1}\left(\bar{X}_{1}\right)\right]$ and $\left[H_{c}^{1}\left(\bar{X}_{2}\right)\right]$ are algebraically dependent in $K_{0}\left(\operatorname{Rep}_{G_{k}} \mathbb{Q}_{l}\right)$. But Theorem $[26$ still shows that the $\mu_{k}\left(\left[X_{i}\right]\right)(i=1,2)$ (and hence the $\left[X_{i}\right]$ themselves) are algebraically independent. The curve $X_{1} \times_{k} K / K$ is not special for any finite extension $k \subset K$ as explained before Theorem 26 and the Jacobians of $X_{1}$ and $X_{2}$ are not isogeneous because they are of different dimensions.

Another consequence of Theorem 26 is that two curves of different genera have algebraically independent classes unless they both become special after some finite extension of the base field. As the referee points out, over $k=\mathbb{C}$ one can easily show that two curves of different genera have algebraically independent classes using a motivic measure based on the Hodge polynomial; see [LL2], 3.4.

Proof of Theorem 26. Since we are allowed to make a finite extension of $k$ we can assume by Theorem 20 that there is an irreducible $G \in \mathbb{Z}\left[T_{1}, T_{2}\right]$ with $G\left(\mu_{k}\left(\left[X_{1}\right]\right), \mu_{k}\left(\left[X_{2}\right]\right)\right)=0$. Writing

$$
f_{i, \nu}:=\partial_{\nu}\left(\mu_{k}\left(\left[X_{i}\right]\right)\right) \in \mathbb{Q}[T], i=1,2, \nu \geq 1,
$$

we have

$$
G\left(f_{1, \nu}, f_{2, \nu}\right)=0 \text { for all } \nu \geq 1 \text {. }
$$

Introducing, for $\nu \geq 1$,

$$
\begin{aligned}
a_{\nu} & :=-\operatorname{tr}\left(F_{k}^{\nu} \mid H_{c}^{1}\left(\bar{X}_{1}\right)\right), \\
b_{\nu} & :=-\operatorname{tr}\left(F_{k}^{\nu} \mid H_{c}^{1}\left(\bar{X}_{2}\right)\right),
\end{aligned}
$$

where $F_{k}$ is the geometric Frobenius of $k=\mathbb{F}_{q}$, say, we have

$$
\begin{gathered}
f_{1, \nu}=1+a_{\nu} T+q^{\nu} T^{2}, \\
f_{2, \nu}=1+b_{\nu} T+q^{\nu} T^{2}
\end{gathered}
$$

because $H_{c}^{0}\left(\bar{X}_{i}\right)=\mathbb{Q}_{l}$ and $H_{c}^{2}\left(\bar{X}_{i}\right)=\mathbb{Q}_{l}(-1)$ and using Proposition 81). We consider

$$
F\left(T_{1}, T_{2}\right):=q\left(T_{2}-T_{1}\right)^{2}-\left(a_{1}-b_{1}\right)\left(a_{1} T_{2}-b_{1} T_{1}\right)+\left(a_{1}-b_{1}\right)^{2} .
$$

The discriminant of $F$, considered as a polynomial in $T_{2}$, equals

$$
4 q\left(a_{1}-b_{1}\right)^{2} T_{1}+\left(a_{1}-b_{1}\right)^{2}\left(a_{1}^{2}-4 q\right) .
$$


The choice of $F$ is made such that we have

$$
\begin{array}{r}
F\left(f_{1,1}, f_{2,1}\right)=F\left(1+a_{1} T+q T^{2}, 1+b_{1} T+q T^{2}\right) \\
=q\left(b_{1}-a_{1}\right)^{2} T^{2}-\left(a_{1}-b_{1}\right)\left(a_{1}+a_{1} b_{1} T+a_{1} q T^{2}\right. \\
\left.-b_{1}-b_{1} a_{1} T-b_{1} q T^{2}\right)+\left(a_{1}-b_{1}\right)^{2} \\
=q\left(b_{1}-a_{1}\right)^{2} T^{2}-\left(a_{1}-b_{1}\right)^{2}\left(1+q T^{2}\right)+\left(a_{1}-b_{1}\right)^{2} \\
=\left(a_{1}-b_{1}\right)^{2}\left(q T^{2}-1-q T^{2}\right)+\left(a_{1}-b_{1}\right)^{2}=0 .
\end{array}
$$

To continue, we make the following additional assumption:

1) $a_{1} \neq b_{1}$ : The prime ideal

$$
P_{1}:=\operatorname{ker}\left(\mathbb{Q}\left[T_{1}, T_{2}\right] \longrightarrow \mathbb{Q}[T], T_{i} \mapsto f_{i, 1}\right)
$$

is proper and non-zero, hence of height 1 in $\mathbb{Q}\left[T_{1}, T_{2}\right]$. By (71) $0 \neq G \in P_{1}$ and $G$ is irreducible. Since $\mathbb{Q}\left[T_{1}, T_{2}\right]$ is factorial we have $P_{1}=(G)$, the principal ideal generated by $G$. As $a_{1} \neq b_{1}$ the discriminant (73) of $F$ is not a square in $\mathbb{Q}\left[T_{1}\right]$, so $F$ is irreducible in $\mathbb{Q}\left[T_{1}, T_{2}\right]$. By (74) we have $F \in P_{1}$, and the same reasoning as for $G$ gives $P_{1}=(F)=(G)$. So there is some $\alpha \in \mathbb{Q}^{*}$ with $F=\alpha G$ from which we

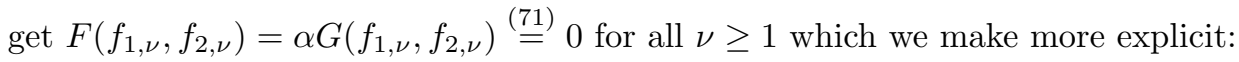

$$
\begin{array}{r}
0=F\left(f_{1, \nu}, f_{2, \nu}\right)=F\left(1+a_{\nu} T+q^{\nu} T^{2}, 1+b_{\nu} T+q^{\nu} T^{2}\right) \\
\stackrel{(72)}{=} q\left(\left(b_{\nu}-a_{\nu}\right) T\right)^{2}-\left(a_{1}-b_{1}\right) \\
\left(a_{1}+a_{1} b_{\nu} T+a_{1} q^{\nu} T^{2}-b_{1}-b_{1} a_{\nu} T-b_{1} q^{\nu} T^{2}\right)+\left(a_{1}-b_{1}\right)^{2} \\
=T^{2}\left(q\left(b_{\nu}-a_{\nu}\right)^{2}-\left(a_{1}-b_{1}\right)\left(a_{1} q^{\nu}-b_{1} q^{\nu}\right)\right)+T\left(-\left(a_{1}-b_{1}\right)\right. \\
\left.\left(a_{1} b_{\nu}-b_{1} a_{\nu}\right)\right)-\left(a_{1}-b_{1}\right)^{2}+\left(a_{1}-b_{1}\right)^{2} \\
=T^{2}\left(q\left(b_{\nu}-a_{\nu}\right)^{2}-q^{\nu}\left(a_{1}-b_{1}\right)^{2}\right)+T\left(\left(a_{1}-b_{1}\right)\left(b_{1} a_{\nu}-a_{1} b_{\nu}\right)\right) .
\end{array}
$$

So for any $\nu \geq 1$ (using $\left.a_{1}-b_{1} \neq 0\right)$ :

$$
\begin{aligned}
q\left(b_{\nu}-a_{\nu}\right)^{2} & =q^{\nu}\left(a_{1}-b_{1}\right)^{2}, \\
b_{1} a_{\nu} & =a_{1} b_{\nu} .
\end{aligned}
$$

Now we assume in addition to $a_{1} \neq b_{1}$ that we also have

1.1) $a_{1} \neq 0$ and $b_{1} \neq 0$ : From (75) we have

$$
b_{\nu}^{2}-2 a_{\nu} b_{\nu}+a_{\nu}^{2}=q^{\nu-1}\left(a_{1}-b_{1}\right)^{2}
$$

into which we substitute (76) to get

$$
a_{\nu}^{2} \frac{b_{1}^{2}}{a_{1}^{2}}-2 a_{\nu}^{2} \frac{b_{1}}{a_{1}}+a_{\nu}^{2}=q^{\nu-1}\left(a_{1}-b_{1}\right)^{2} .
$$

Multiplying this by $a_{1}^{2}$ gives

$$
a_{\nu}^{2}\left(b_{1}^{2}-2 a_{1} b_{1}+a_{1}^{2}\right)=q^{\nu-1}\left(a_{1}-b_{1}\right)^{2} a_{1}^{2},
$$

and cancelling $\left(a_{1}-b_{1}\right)^{2} \neq 0$ we obtain

$$
a_{\nu}^{2}=q^{\nu-1} a_{1}^{2} \text { for } \nu \geq 1 .
$$


We have $a_{\nu}^{2}=\operatorname{tr}\left(F_{k}^{\nu} \mid H_{c}^{1}\left(\bar{X}_{1}\right)^{\otimes 2}\right)$, hence

$$
\begin{aligned}
\operatorname{det}\left(1-F_{k} t \mid H_{c}^{1}\left(\bar{X}_{1}\right)^{\otimes 2}\right) & =\exp \left(-\sum_{\nu \geq 1} a_{\nu}^{2} \frac{t^{\nu}}{\nu}\right) \\
\stackrel{(777)}{=} \exp \left(-\sum_{\nu \geq 1} q^{\nu-1} a_{1}^{2} \frac{t^{\nu}}{\nu}\right) & =\exp \left(q^{-1} a_{1}^{2}\left(\sum_{\nu \geq 1}-\frac{(q t)^{\nu}}{\nu}\right)\right) \\
& =(1-q t)^{a_{1}^{2} / q} .
\end{aligned}
$$

The first equality in (78) is $\left[\mathrm{H}\right.$, Appendix C, Lemma 4.1. We denote by $g\left(X_{i}\right)$ the genus of $X_{i}$. As $\operatorname{dim}_{\mathbb{Q}_{l}} H_{c}^{1}\left(\bar{X}_{i}\right)=2 g\left(X_{i}\right)$ we obtain from (78) by comparing degrees:

$$
a_{1}^{2}=4 g\left(X_{1}\right)^{2} q .
$$

Denoting by $\alpha_{1}, \ldots, \alpha_{2 g\left(X_{1}\right)}$ the eigenvalues of $F_{k}$ acting on $H_{c}^{1}\left(\bar{X}_{1}\right)$ considered as complex numbers via some isomorphism $\overline{\mathbb{Q}_{l}} \stackrel{\sim}{\rightarrow} \mathbb{C}$, we have $\left|\alpha_{i}\right|=q^{1 / 2}$ and thus

$$
\left|a_{1}^{2}\right|=\left|-\sum_{i=1}^{2 g\left(X_{1}\right)} \alpha_{i}\right|^{2} \leq\left(2 g\left(X_{1}\right) q^{1 / 2}\right)^{2}=4 g\left(X_{1}\right)^{2} q .
$$

By (79) this upper bound is in fact an equality which forces all $\alpha_{i}$ to be equal to either $+q^{1 / 2}$ or $-q^{1 / 2}$. After possibly a quadratic extension of $k$ we will have all $\alpha_{i}$ equal to $+q^{1 / 2}$ and then $X_{1} / k$ will be special by definition. As the additional assumptions we have made so far, namely that $a_{1} \neq b_{1}$ and $a_{1}, b_{1} \neq 0$, are symmetric in $X_{1}$ and $X_{2}$ the same conclusion holds for $X_{2} / k$, and we are in case 1) of Theorem 26. Now we assume:

1.2) $a_{1}=0$ : We then have, as we still assume that $a_{1} \neq b_{1}$, that $b_{1} \neq 0$ and (76) implies that $a_{\nu}=0$ for all $\nu \geq 1$, hence $H_{c}^{1}\left(\bar{X}_{1}\right)=0$ and $X_{1} \simeq \mathbb{P}^{1}$. Note that there are no non-trivial $\bar{k} / k$-forms of $\mathbb{P}^{1}$ because the Brauer group of a finite field is trivial. From (75) we then get $b_{\nu}^{2}=q^{\nu-1} b_{1}^{2}$, and the same argument as in case 1.1) (cf. (77)) shows that $X_{2}$ becomes special after at most a quadratic extension. So we are again in case 1 ) of Theorem 26 .

1.3) $b_{1}=0$ : this case is symmetric to the case 1.2$)$ above.

There is one case left to be considered.

2) $a_{1}=b_{1}$ : We now have $f_{1,1}=f_{2,1}$ and $F:=T_{1}-T_{2}$ satisfies $F\left(f_{1,1}, f_{2,1}\right)=0$. By the same arguments as in case 1$)$ we get $F\left(f_{1, \nu}, f_{2, \nu}\right)=0$ for all $\nu \geq 1$, i.e.

$$
0=F\left(1+a_{\nu} T+q^{\nu} T^{2}, 1+b_{\nu} T+q^{\nu} T^{2}\right)=\left(a_{\nu}-b_{\nu}\right) T,
$$

so $a_{\nu}=b_{\nu}$ for all $\nu \geq 1$ from which we get

$$
\operatorname{det}\left(1-F_{k} t \mid H_{c}^{1}\left(\bar{X}_{1}\right)\right)=\operatorname{det}\left(1-F_{k} t \mid H_{c}^{1}\left(\bar{X}_{2}\right)\right)
$$

which is equivalent to the Jacobians of $X_{1}$ and $X_{2}$ being $k$-isogeneous [T], Theorem $1, \mathrm{c} 1) \Leftrightarrow \mathrm{c} 2$ ), and hence we are in case 2) of Theorem 26 .

Remark. We conserve the notations of the above proof. The simplest case in which Theorem 26] does not guarantee the algebraic independence of the classes of two curves in $K_{0}\left(\operatorname{Var}_{k}\right)$ is if $X_{1}=\mathbb{P}^{1}$ and $X_{2}=E$ is a super-singular elliptic curve. Let $k$ be so large that $X_{2} / k$ is special and put $q:=|k|$. Then $F:=q\left(T_{2}-T_{1}\right)^{2}-4 q T_{1}+4 q$ satisfies

$$
F\left(\mu_{k}\left(\left[X_{1}\right]\right), \mu_{k}\left(\left[X_{2}\right]\right)\right)=0 .
$$


In fact, we have

$$
\begin{aligned}
f_{1, \nu}=1+q^{\nu} T^{2} & =\partial_{\nu}\left(\mu_{k}\left(\left[X_{1}\right]\right)\right), \\
f_{2, \nu}=1-2 q^{\nu / 2} T+q^{\nu} T^{2} & =\partial_{\nu}\left(\mu_{k}\left(\left[X_{2}\right]\right)\right)
\end{aligned}
$$

and compute

$$
\begin{aligned}
F\left(\partial_{\nu}\left(\mu_{k}\left(\left[X_{1}\right]\right)\right), \partial_{\nu}\left(\mu_{k}\left(\left[X_{2}\right]\right)\right)\right) & =q\left(-2 q^{\nu / 2} T\right)^{2}-4 q\left(1+q^{\nu} T^{2}\right)+4 q \\
=4 q^{\nu+1} T^{2}-4 q^{\nu+1} T^{2}-4 q+4 q & =0,
\end{aligned}
$$

for all $\nu \geq 1$, hence (80) follows. On the other hand

$$
F\left(\left[X_{1}\right],\left[X_{2}\right]\right)=\left[Z^{+}\right]-\left[Z^{-}\right] \text {in } K_{0}\left(\operatorname{Var}_{k}\right),
$$

where $Z^{+}$and $Z^{-}$are the (non-connected) proper, smooth varieties

$$
\begin{array}{r}
Z^{+}=\left(E^{2}\right)^{\sqcup q} \sqcup\left(\left(\mathbb{P}^{1}\right)^{2}\right)^{\sqcup q} \sqcup \operatorname{Spec}(k)^{\sqcup 4 q}, \\
Z^{-}=\left(E \times \mathbb{P}^{1}\right)^{\sqcup 2 q} \sqcup\left(\mathbb{P}^{1}\right)^{\sqcup 4 q} .
\end{array}
$$

The sign $\sqcup$ means disjoint union of schemes. I cannot decide whether $\left[Z^{+}\right]$and $\left[Z^{-}\right]$are equal (i.e. whether $F\left(\left[X_{1}\right],\left[X_{2}\right]\right)=0$ ), but I suspect not and even more, that $\left[\mathbb{P}^{1}\right]$ and $[E]$ should be algebraically independent. Note at least that $Z^{+} \nsucceq Z^{-}$, e.g. $Z^{+}$has isolated points but $Z^{-}$does not.

This illustrates that our methods can only give sufficient conditions for varieties to be algebraically independent in the Grothendieck ring of varieties, but we have no idea how to produce interesting relations in $K_{0}\left(\operatorname{Var}_{k}\right)$.

\section{ACKNOWLEDGEMENTS}

I would like to thank my advisor C. Deninger for constant support and encouragement during the preparation of my thesis, the main results of which are presented here. Furthermore, I would like to thank H. Frommer, U. Jannsen, M. Kisin, B. Moonen, D. Roessler, C. Serpé and C. Soulé for useful discussions and the referee for a very thorough report.

\section{REFERENCES}

[Algèbre] N. Bourbaki, Algèbre, Chapitre 8, Hermann, Paris, 1958. MR0098114 (20:4576)

[A] Y. André, Motifs de dimensione finie, Séminaire Bourbaki, 56ème année, 2003-2004, no 929. MR.2167204

[Bi] F. Bittner, The universal Euler characteristic for varieties of characteristic zero, Compos. Math. 140 (2004), no. 4, 1011-1032. MR2059227(2005d:14031)

[Bo] A. Borel, Linear algebraic groups, W.A. Benjamin, Inc., New York, Amsterdam, 1969. MR0251042 (40:4273)

[BDS] I. Bouw, C. Diem, J. Scholten, Ordinary elliptic curves of high rank over $\overline{\mathbb{F}_{p}}(x)$ with constant $j$-invariant, Manuscripta Math. 114 (2004), 487-501. MR2081948 (2005e:11069)

$[\mathrm{DH}] \quad H$. Davenport, H. Hasse, Die Nullstellen der Kongruenzzetafunktion in gewissen zyklischen Fällen, J. Reine Angew. Math. 172 (1935), 151-182.

[D1] P. Deligne, Théorie de Hodge. II, Inst. Hautes Études Sci. Publ. Math. no. 40 (1971), 5-57. MR0498551 (58:16653a)

[D2] P. Deligne, Catégories tannakiennes, The Grothendieck Festschrift, Vol. II, 111-195, Progr. Math. 87, Birkhäuser, Boston, 1990. MR1106898 (92d:14002)

[D3] P. Deligne, La conjecture de Weil. II, Inst. Hautes Études Sci. Publ. Math. no. 52 (1980), 137-252. MR0601520 (83c:14017)

[DMOS] P. Deligne, J. Milne, A. Ogus, K. Shih, Hodge cycles, Motives and Shimura Varieties, LNM 900, Springer, 1989, 101-228. MR0654325 (84m:14046)

[DG] M. Demazure, P. Gabriel, Groupes algébriques. Tome I, North-Holland Publishing Co., Amsterdam, 1970. MR0302656 (46:1800) 
[G] A. Grothendieck, Letter to J-P. Serre (dated 16.8.1964), in Correspondance Grothendieck-Serre, P. Colmez, J-P. Serre (eds.), Documents Mathématiques, Soc. Math. France, 2001. MR1942134 (2003k:14002)

[H] R. Hartshorne, Algebraic geometry, Graduate Texts in Mathematics, No. 52, Springer, New York, 1977. MR0463157 (57:3116)

[J] U. Jannsen, Mixed motives and algebraic $K$-theory, LNM 1400, Springer, 1990. MR 1043451 (91g:14008)

[K] M. Kapranov, The elliptic curve in the S-duality theory and Eisenstein-series for KacMoody groups, math.AG/0001005.

[Ka] N. Katz, Review of $l$-adic cohomology, in Motives, Proc. Symp. Pure Math. 55, part 1, 21-30. MR.1265520 (95j:14021)

[Ko] J. Kollár, Conics in the Grothendieck ring, Adv. Math. 198 (2005), 27-35. MR2183248

[LL1] M. Larsen, V. Lunts, Rationality criteria for motivic zeta functions, Compos. Math. 140, no. 6 (2004), 1537-1560. MR2098401 (2005k:14045)

[LL2] M. Larsen, V. Lunts, Motivic measures and stable birational geometry, Mosc. Math. J. 3, no. 1 (2003), 85-95. MR1996804(2005a:14026)

[Lo] E. Looijenga, Motivic measures, Séminaire Bourbaki, Vol. 1999/2000. Astérisque No. 276 (2002), 267-297. MR:1886763 (2003k:14010)

[Ma] J. Manin, Theory of commutative formal groups over fields of finite characteristic, Uspeki Mat. Nauk 18 (1963), no. 6, 3-90. MR0157972 (28:1200)

[Mi1] J. Milne, Motives over finite fields, in Motives, Proc. Symp. Pure Math. 55, part 1, 401-459. MR.1265538 (95g:11053)

[Mi2] J. Milne, Étale cohomology, Princeton University Press, Princeton N.J., 1980. MR0559531 (81j:14002)

[P] B. Poonen, The Grothendieck ring of varieties is not a domain, Math. Res. Letters 9 (2002), no. 4, 493-497. MR1928868 (2003g:14010)

[Q] D. Quillen, Higher algebraic K-theory. I, LNM 341, 85-147, Springer, Berlin, 1973. MR0338129 (49:2895)

[R] K. Ribet, Galois action on division points of Abelian varieties with real multiplications, Amer. J. Math. 98 (1976), no. 3, 751-804. MR0457455 (56:15660)

[RS] J. Rosser, L. Schoenfeld, Approximate formulas for some functions of prime numbers, Illinois J. Math. 6 (1962), 64-94. MR0137689 (25:1139)

[S1] J-P. Serre, Groupes de Grothendieck des schémas en groupes réductifs déployés, Inst. Hautes Études Sci. Publ. Math. no. 34 (1968), 37-52. MR0231831 (38:159)

[S2] J-P. Serre, Un exemple de série de Poincaré non rationnelle, Proc. Nederland Acad. Sci. 82 (1979), 469-471. MR0554140 (81a:55014)

[S3] J-P. Serre, Linear representations of finite groups, Graduate Texts in Mathematics, Vol. 42, Springer, New York, 1977. MR0450380 (56:8675)

[S4] J-P. Serre, Groupes algébriques associés aux modules de Hodge-Tate, Journées de Géométrie Algébriques de Rennes, Vol. III, 155-188, Astérisque 65, Soc. Math. France, 1979. MR0563476 (81j:14027)

[S5] J-P. Serre, Letter to K. Ribet dated 1.1.1981 and 29.1.1981 in Collected Papers IV, no. 133, Springer, 2003. MR.1730973 (2001e:01037)

[T] J. Tate, Endomorphisms of abelian varieties over finite fields, Invent. Math. 2 (1966), 134-144. MR 0206004 (34:5829)

[Y] T. Yamada, On the Davenport-Hasse curves, J. Math. Soc. Japan, Vol. 20, nos. 1-2 (1968), 403-410. MR0225782(37:1375)

NWF I- Mathematik, Universität Regensburg, Universitätsstrasse 31, 93053 RegensBURG, Germany 